\title{
Matching the heavy-quark fields in QCD and HQET at four loops
}

\author{
Andrey G. Grozin $\odot,{ }^{1,2, *}$ Peter Marquard $\odot,{ }^{3, \dagger}$ Alexander V. Smirnov $\odot,{ }^{4,5, \$}$ \\ Vladimir A. Smirnov $\odot^{6,5,8}$ and Matthias Steinhauser $\circledast^{7, \|}$ \\ ${ }^{1}$ Budker Institute of Nuclear Physics, 630090 Novosibirsk, Russia \\ ${ }^{2}$ Novosibirsk State University, 630090 Novosibirsk, Russia \\ ${ }^{3}$ Deutsches Elektronen-Synchrotron, DESY, 15738 Zeuthen, Germany \\ ${ }^{4}$ Research Computing Center, Moscow State University, 119991 Moscow, Russia \\ ${ }^{5}$ Moscow Center for Fundamental and Applied Mathematics, 119992 Moscow, Russia \\ ${ }^{6}$ Skobeltsyn Institute of Nuclear Physics of Moscow State University, 119991 Moscow, Russia \\ ${ }^{7}$ Institut für Theoretische Teilchenphysik, Karlsruher Institut für Technologie (KIT), \\ 76128 Karlsruhe, Germany
}

(Received 4 June 2020; accepted 3 August 2020; published 8 September 2020)

\begin{abstract}
The QCD/HQET matching coefficient for the heavy-quark field is calculated up to four loops. It must be finite; this requirement produces analytical results for some terms in the four-loop on-shell heavy-quark field renormalization constant which were previously only known numerically. The effect of a nonzero lighter-flavor mass is calculated up to three loops. A class of on-shell integrals with two masses is analyzed in detail. By specifying our result to QED, we obtain the relation between the electron field and the BlochNordsieck field with four-loop accuracy.
\end{abstract}

DOI: 10.1103/PhysRevD.102.054008

\section{INTRODUCTION}

Some classes of QCD problems with a single heavy quark can be examined in a simpler effective theory, the so-called heavy quark effective theory (HQET, see, e.g., [1-3]). Let us consider QCD with a single heavy flavor $Q$ and $n_{l}$ light flavors $\left(n_{f}=n_{l}+n_{h}, n_{h}=1\right)$. The heavy-quark momentum can be decomposed as $p=M v+k$, where $M$ is the on-shell $Q$ mass, and $v$ is some reference 4-velocity $\left(v^{2}=1\right)$. In the case of QED, it is called Bloch-Nordsieck effective theory [4].

In the effective theory, the heavy quark (respectively lepton) is represented by the field $h_{v}$. The $\overline{\mathrm{MS}}$ renormalized fields $Q(\mu)$ and $h_{v}(\mu)$ are related by [5]

$Q(\mu)=e^{-i M v x}\left[\sqrt{z(\mu)}\left(1+\frac{\not D_{\perp}}{2 M}\right) h_{v}(\mu)+\mathcal{O}\left(\frac{1}{M^{2}}\right)\right]$,

where $D_{\perp}^{\mu}=D^{\mu}-v^{\mu} v \cdot D$, and the matching coefficient is given by

\footnotetext{
*A.G.Grozin@inp.nsk.su peter.marquard@desy.de *asmirnov80@gmail.com smirnov@theory.sinp.msu.ru

"matthias.steinhauser@kit.edu
}

Published by the American Physical Society under the terms of the Creative Commons Attribution 4.0 International license. Further distribution of this work must maintain attribution to the author(s) and the published article's title, journal citation, and DOI. Funded by SCOAP ${ }^{3}$.

$$
z(\mu)=\frac{Z_{h}\left(\alpha_{s}^{\left(n_{l}\right)}(\mu), \xi^{\left(n_{l}\right)}(\mu)\right) Z_{Q}^{\mathrm{os}}\left(g_{0}^{\left(n_{f}\right)}, \xi_{0}^{\left(n_{f}\right)}\right)}{Z_{Q}\left(\alpha_{s}^{\left(n_{f}\right)}(\mu), \xi^{\left(n_{f}\right)}(\mu)\right) Z_{h}^{\mathrm{os}}\left(g_{0}^{\left(n_{l}\right)}, \xi_{0}^{\left(n_{l}\right)}\right)} .
$$

Here $Z_{Q}^{\text {os }}$ and $Z_{h}^{\text {os }}$ are the on-shell field renormalization constants (they depend on the corresponding bare couplings and bare gauge-fixing parameters), and $Z_{Q}$ and $Z_{h}$ are the $\overline{\mathrm{MS}}$ renormalization constants. The covariant-gauge fixing parameter is defined in such a way that the bare gluon propagator is given by $\left(g_{\mu \nu}-\xi_{0} p_{\mu} p_{\nu} / p^{2}\right) / p^{2}$; it is renormalized by the gluon-field renormalization constant: $1-\xi_{0}=Z_{A}\left(\alpha_{s}(\mu), \xi(\mu)\right)(1-\xi(\mu))$. The $1 / M$ correction in (1) is fixed by reparametrization invariance [6].

The $\overline{\mathrm{MS}}$ renormalized matching coefficient is obviously finite at $\varepsilon \rightarrow 0$, because it relates the off-shell renormalized propagators in the two theories, which are both finite. The ultraviolet divergences cancel in the ratios $Z_{Q} / Z_{Q}^{\text {os }}$ and $Z_{h} / Z_{h}^{\text {os }}$, because they relate renormalized fields; the infrared divergences cancel in $Z_{Q}^{\text {os }} / Z_{h}^{\text {os }}$, because HQET is constructed to reproduce the infrared behavior of QCD; the $\overline{\mathrm{MS}}$ renormalization constants $Z_{Q}$ and $Z_{h}$ (purely offshell quantities) are infrared finite. If we assume that all light flavors are massless we have $Z_{h}^{\text {os }}=1$ : all loop corrections vanish because they contain no scale, ultraviolet and infrared divergences of $Z_{h}^{\text {os }}$ mutually cancel. Taking light-quark masses $m_{i}$ into account produces corrections suppressed by powers of $m_{i} / M$, see Sec. III.

The matching coefficient satisfies the renormalizationgroup equation 


$$
\begin{aligned}
& \frac{d \log z(\mu)}{d \log \mu} \\
& \quad=\gamma_{h}\left(\alpha_{s}^{\left(n_{l}\right)}(\mu), \xi^{\left(n_{l}\right)}(\mu)\right)-\gamma_{Q}\left(\alpha_{s}^{\left(n_{f}\right)}(\mu), \xi^{\left(n_{f}\right)}(\mu)\right),
\end{aligned}
$$

where the anomalous dimensions are defined as $\gamma_{i}=$ $d \log Z_{i} / d \log \mu(i=Q, h)$. It is sufficient to obtain the initial condition $z\left(\mu_{0}\right)$ for some scale $\mu_{0} \sim M ; z(\mu)$ for other renormalization scales $\mu$ can be found by solving Eq. (3). We choose to present the result for $\mu_{0}=M$.

The heavy-quark field matching coefficient $z(\mu)$ has been calculated up to three loops [5]. When the matching coefficient is used within a quantity containing $1 / \varepsilon$ divergences, terms with positive powers of $\varepsilon$ in $z(\mu)$ are needed; such terms were not given in [5]. We present the four-loop result in Sec. II. Power corrections due to lighterflavor masses up to three loops are obtained in Sec. III. The QED result, i.e., the four-loop relation between the lepton field and the Bloch-Nordsieck field, is discussed in Sec. IV. In Appendix A we provide analytic results for the decoupling coefficients for the strong coupling constant and the gluon field up to three-loop order including linear $\varepsilon$ terms. Appendix B contains a detailed analysis of a class of onshell integrals with two masses. It allows us, in particular, to obtain exact results for the three-loop term in the $\overline{\mathrm{MS}}$-onshell mass relation with a closed massless and a closed lighter-flavor massive fermion loop (previously this term was only known as a truncated series in this mass ratio).

\section{THE QCD AND HQET HEAVY-QUARK FIELDS}

If we assume that all light flavors are massless, then (2) gives

$$
\begin{aligned}
\log z(\mu)= & \log Z_{Q}^{\mathrm{os}}\left(g_{0}^{\left(n_{f}\right)}, \xi_{0}^{\left(n_{f}\right)}\right) \\
& -\log Z_{Q}\left(\alpha_{s}^{\left(n_{f}\right)}(\mu), \xi^{\left(n_{f}\right)}(\mu)\right) \\
& +\log Z_{h}\left(\alpha_{s}^{\left(n_{l}\right)}(\mu), \xi^{\left(n_{l}\right)}(\mu)\right) .
\end{aligned}
$$

The on-shell heavy-quark field renormalization constant $Z_{Q}^{\text {os }}$ depends on the bare coupling $g_{0}^{\left(n_{f}\right)}$, the bare gauge parameter $\xi_{0}^{\left(n_{f}\right)}$ and the on-shell mass $M$ :

$$
\begin{aligned}
& Z_{Q}^{\mathrm{os}}=1+\sum_{L=1}^{\infty}\left(4 \frac{\left(g_{0}^{\left(n_{f}\right)}\right)^{2} M^{-2 \varepsilon}}{(4 \pi)^{d / 2}} e^{-\gamma_{E} \varepsilon}\right)^{L} Z_{L} \\
& Z_{L}=\sum_{n=0}^{\infty} Z_{L, n}\left(\xi_{0}^{\left(n_{f}\right)}\right) \varepsilon^{n-L}
\end{aligned}
$$

The two-loop expression is known exactly in $\varepsilon$ [7]; it contains a single nontrivial master integral, further terms of its $\varepsilon$ expansion are presented in [8,9]. The three-loop term has been calculated in $[10,11]$. At four loops, the terms with $n_{l}^{3}$ and $n_{l}^{2}$ are known analytically [12], and the remaining ones numerically [13]. Recently the QED-like color structures $C_{F}^{4}, C_{F}^{3} T_{F} n_{h}, C_{F}^{2}\left(T_{F} n_{h}\right)^{2}, C_{F}\left(T_{F} n_{h}\right)^{3}, d_{F F} n_{h}$ have been calculated analytically [14]. Here and below we use the notation

$$
d_{F F}=\frac{d_{F}^{a b c d} d_{F}^{a b c d}}{N_{F}}, \quad d_{F A}=\frac{d_{F}^{a b c d} d_{A}^{a b c d}}{N_{F}},
$$

where $N_{R}=\operatorname{Tr} \mathbf{1}_{R} \quad($ with $\quad R=F), \quad d_{R}^{a b c d}=\operatorname{Tr} t_{R}^{(a} t_{R}^{b} t_{R}^{c} t_{R}^{d)}$ (with $R=F$ or $A$ ), and the round brackets mean symmetrization (for $S U\left(N_{c}\right)$ gauge group $d_{F F}=\left(N_{c}^{2}-1\right.$ ) $\left.\left(N_{c}^{4}-6 N_{c}^{2}+18\right) /\left(96 N_{c}^{3}\right), \quad d_{F A}=\left(N_{c}^{2}-1\right)\left(N_{c}^{2}+6\right) / 48\right)$. This result contains the same master integrals as the electron $g-2[15,16]$. In [15] they have been calculated numerically to 1100 digits, and analytical expressions have been reconstructed using PSLQ. In the case of the light-bylight contribution $d_{F F} n_{h}$ the results contain $\varepsilon^{0}$ terms of 6 master integrals (known numerically to 1100 digits); all the remaining constants are completely expressed via known transcendental numbers. (Note that the definition of the constant $t_{63}$ is missing in the journal article [14]; it is included in the version $\mathrm{v} 3$ of the arXiv publications.)

The $\overline{\mathrm{MS}}$ quark-field anomalous dimension $\gamma_{q}$ (and hence $\log Z_{Q}$ ) is well known [17-20]. The HQET field anomalous dimension $\gamma_{h}$ (and hence $\log Z_{h}$ ) is known at three loops [10,21]. At four loops, some color structures are known analytically: $C_{F}\left(T_{F} n_{l}\right)^{3}$ [22], $C_{F}^{2}\left(T_{F} n_{l}\right)^{2}$ [23,24], $C_{F} C_{A}\left(T_{F} n_{l}\right)^{2}$ [13], $C_{F}^{3} T_{F} n_{l}$ [25], $d_{F F} n_{l}$ [26], $C_{F}^{2} C_{A} T_{F} n_{l}$ and $C_{F} C_{A}^{2} T_{F} n_{l}$ [27]; $C_{F} C_{A}^{3}$ and $d_{F A}$ are known numerically [13].

We need to express the three terms in (4) in terms of the same set of variables, for which we choose $\alpha_{s}^{\left(n_{f}\right)}(\mu)$ and $\xi^{\left(n_{f}\right)}(\mu)$. Expressing $g_{0}^{\left(n_{f}\right)}$ and $\xi_{0}^{\left(n_{f}\right)}$ via these variables is straightforward, since the three-loop renormalization constants in QCD are well known. Expressing $\alpha_{s}^{\left(n_{l}\right)}(\mu)$ and $\xi^{\left(n_{l}\right)}(\mu)$ via the $n_{f}$-flavor quantities requires decoupling relations up to $\mathcal{O}(\varepsilon)$ at three loops. For convenience we present explicit results in Appendix A.

The resulting matching coefficient $z(M)$ must be finite at $\varepsilon \rightarrow 0$. This requirement together with the known results for $Z_{Q}$ and $Z_{h}$ leads to analytical expressions for the fourloop coefficients $Z_{4,0}, Z_{4,1}$, and $Z_{4,2}$ in (5) as well as for $Z_{4,3}$, except two color structures $C_{F} C_{A}^{3}$ and $d_{F A}$ where the corresponding terms in $\gamma_{h}$ are not known analytically. The analytic results are presented in the Tables I and II. We refrain from showing results for the $n_{l}^{2}$ and $n_{l}^{3}$ terms, which are already known since a few years [12]. Furthermore, we have introduced $a_{n}=\mathrm{Li}_{n}(1 / 2)$ (in particular $a_{1}=\log 2$ ); $\zeta_{n}$ denotes the Riemann zeta function and $\xi_{0}=\xi_{0}^{\left(n_{f}\right)}$. Analytical results for the color structures $C_{F}^{4}, C_{F}^{3} T_{F} n_{h}$, $C_{F}^{2}\left(T_{F} n_{h}\right)^{2}, C_{F}\left(T_{F} n_{h}\right)^{3}, d_{F F} n_{h}$ were recently obtained [14]. They agree with the expressions given in Tables I and II. Numerical results for these coefficients are given in 
TABLE I. Coefficients $Z_{4, n}$ of the $1 / \varepsilon^{4,3,2}$ terms entering the four-loop result $Z_{4}$ in Eq. (5). Note that the color structures $d_{F F} n_{l}, d_{F F} n_{h}$, $d_{F A}$ have zero coefficients.

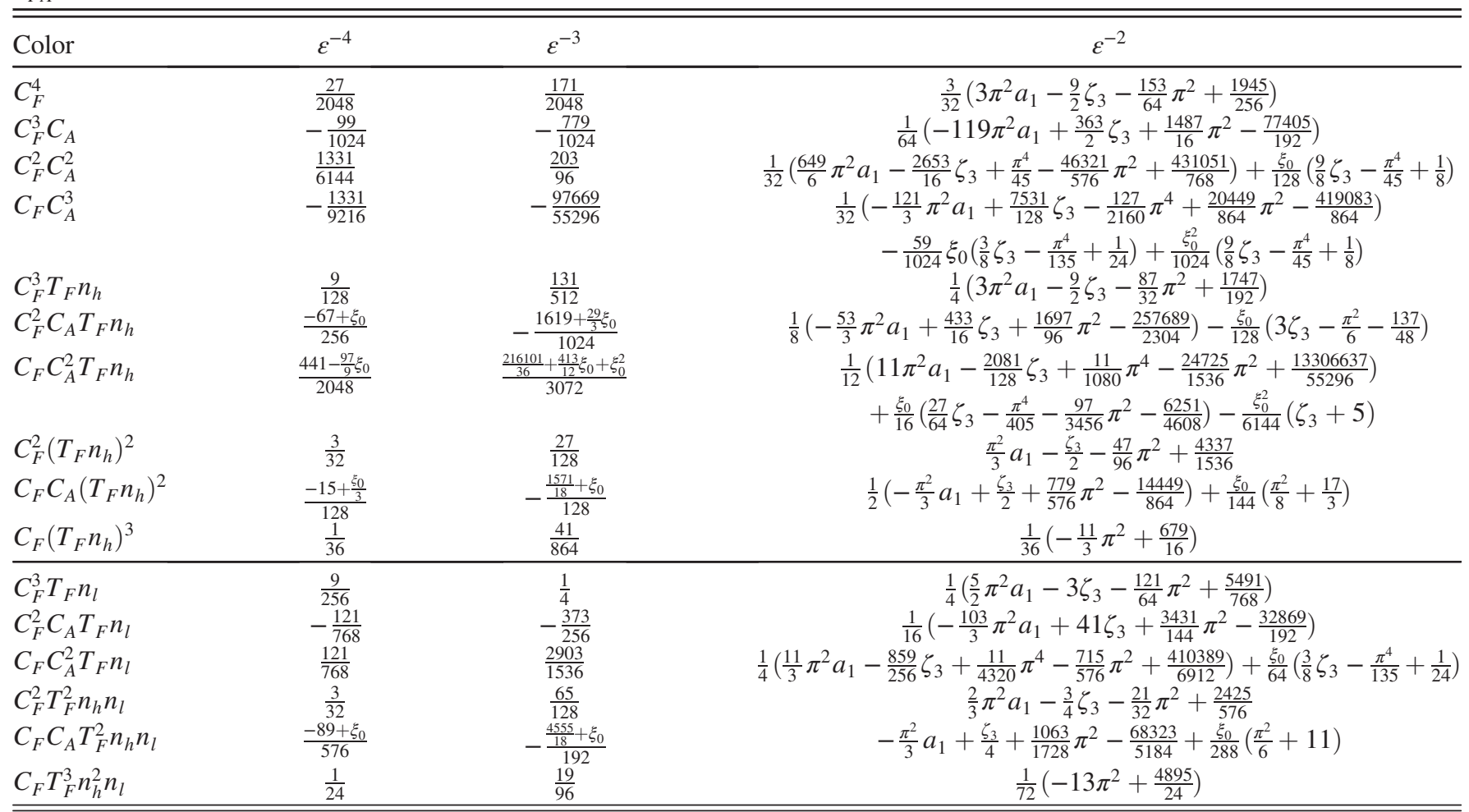

TABLE II. Coefficients $Z_{4,3}$ of the $1 / \varepsilon$ term entering the four-loop result $Z_{4}$ in Eq. (5). Note that the color structures $C_{F} C_{A}^{3}$ and $d_{F A}$ are not known analytically.

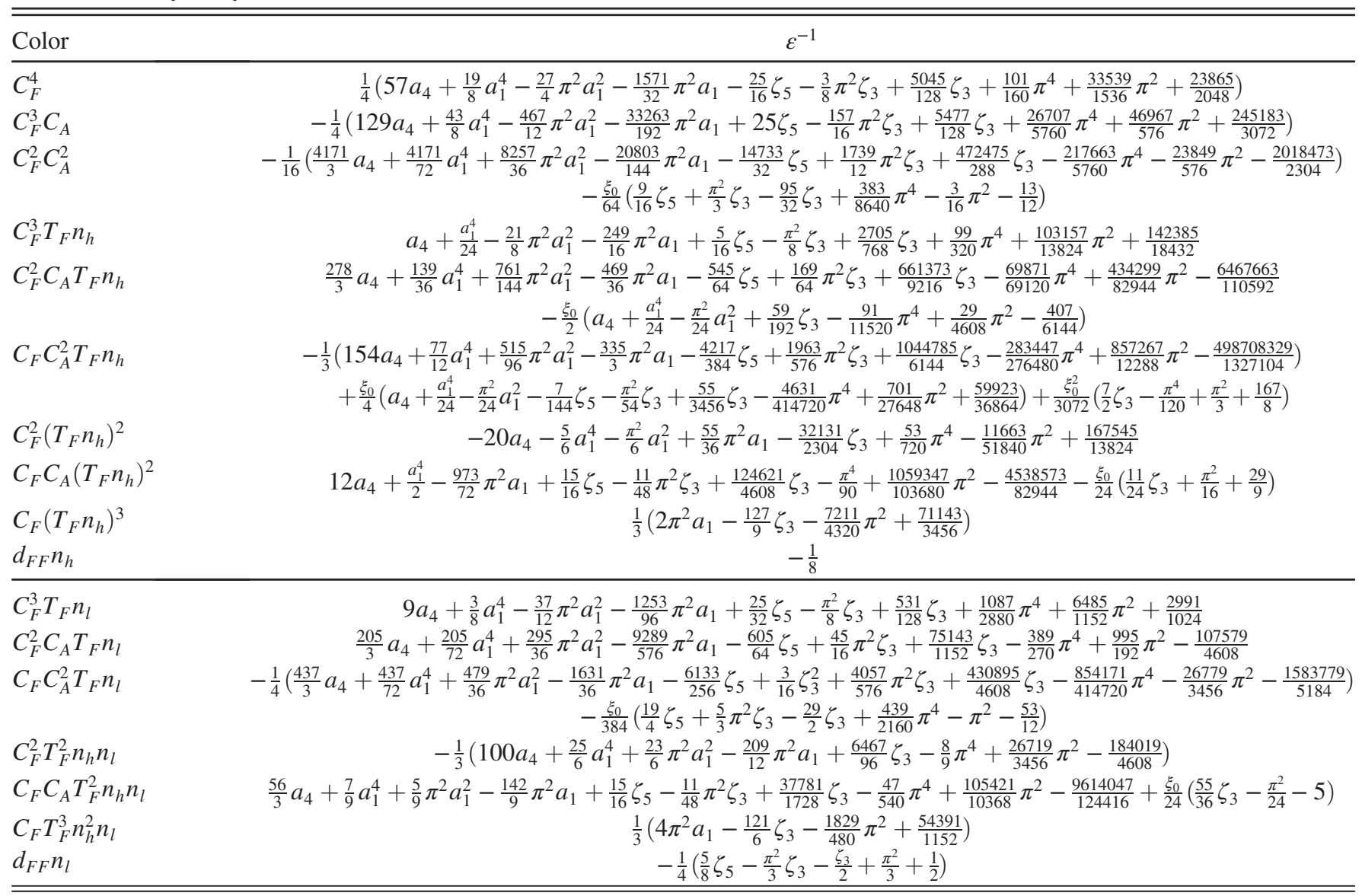


the Tables V, VI, and VII of Ref. [13]. Good agreement is found.

Using the matching coefficient $z(\mu)$ together with quantities which contains $1 / \varepsilon$ divergences, terms with positive powers of $\varepsilon$ are needed. In order to get the finite four-loop contribution, we need the $\alpha_{s}^{L}$ term in $z(\mu)$ expanded up to $\varepsilon^{4-L}$. Our result for $\mu=M$ is given by

$$
\begin{aligned}
& z(M)=1-\frac{\alpha_{s}}{\pi} C_{F}\left[1+\varepsilon\left(\frac{\pi^{2}}{16}+2\right)-\varepsilon^{2}\left(\frac{\zeta_{3}}{4}-\frac{\pi^{2}}{12}-4\right)-\varepsilon^{3}\left(\frac{\zeta_{3}}{3}-\frac{3}{640} \pi^{4}-\frac{\pi^{2}}{6}-8\right)+\mathcal{O}\left(\varepsilon^{4}\right)\right] \\
& +\left(\frac{\alpha_{s}}{\pi}\right)^{2} C_{F}\left\{C_{F}\left(\pi^{2} a_{1}-\frac{3}{2} \zeta_{3}-\frac{13}{16} \pi^{2}+\frac{241}{128}\right)-\frac{C_{A}}{2}\left(\pi^{2} a_{1}-\frac{3}{2} \zeta_{3}-\frac{5}{8} \pi^{2}+\frac{1705}{192}\right)\right. \\
& -\frac{T_{F} n_{h}}{3}\left(\pi^{2}-\frac{947}{96}\right)+\frac{T_{F} n_{l}}{12}\left(\pi^{2}+\frac{113}{8}\right) \\
& +\varepsilon\left[-C_{F}\left(24 a_{4}+a_{1}^{4}+2 \pi^{2} a_{1}^{2}-\frac{23}{4} \pi^{2} a_{1}+\frac{147}{8} \zeta_{3}-\frac{7}{20} \pi^{4}+\frac{347}{128} \pi^{2}+\frac{557}{256}\right)\right. \\
& +C_{A}\left(12 a_{4}+\frac{a_{1}^{4}}{2}+\pi^{2} a_{1}^{2}-\frac{23}{8} \pi^{2} a_{1}+\frac{129}{16} \zeta_{3}-\frac{7}{40} \pi^{4}+\frac{769}{1152} \pi^{2}-\frac{9907}{768}\right) \\
& \left.+T_{F} n_{h}\left(2 \pi^{2} a_{1}-7 \zeta_{3}-\frac{445}{288} \pi^{2}+\frac{17971}{1728}\right)+T_{F} n_{l}\left(\zeta_{3}+\frac{127}{288} \pi^{2}+\frac{851}{192}\right)\right] \\
& +\varepsilon^{2}\left[-C_{F}\left(144 a_{5}+138 a_{4}-\frac{6}{5} a_{1}^{5}+\frac{23}{4} a_{1}^{4}-4 \pi^{2} a_{1}^{3}+\frac{23}{2} \pi^{2} a_{1}^{2}+\frac{13}{15} \pi^{4} a_{1}-\frac{41}{2} \pi^{2} a_{1}\right.\right. \\
& \left.-\frac{609}{4} \zeta_{5}-\frac{11}{4} \pi^{2} \zeta_{3}+\frac{2061}{32} \zeta_{3}-\frac{1555}{1536} \pi^{4}+\frac{8947}{768} \pi^{2}-\frac{1817}{512}\right) \\
& +C_{A}\left(72 a_{5}+69 a_{4}-\frac{3}{5} a_{1}^{5}+\frac{23}{8} a_{1}^{4}-2 \pi^{2} a_{1}^{3}+\frac{23}{4} \pi^{2} a_{1}^{2}+\frac{13}{30} \pi^{4} a_{1}-\frac{41}{4} \pi^{2} a_{1}\right. \\
& \left.-\frac{609}{8} \zeta_{5}-\frac{11}{8} \pi^{2} \zeta_{3}+\frac{7595}{288} \zeta_{3}-\frac{14359}{23040} \pi^{4}+\frac{6367}{2304} \pi^{2}-\frac{79225}{1536}\right) \\
& -T_{F} n_{h}\left(48 a_{4}+2 a_{1}^{4}+4 \pi^{2} a_{1}^{2}-\frac{19}{2} \pi^{2} a_{1}+\frac{2405}{72} \zeta_{3}-\frac{93}{320} \pi^{4}+\frac{8605}{1728} \pi^{2}-\frac{422747}{10368}\right) \\
& \left.\left.+\frac{T_{F} n_{l}}{24}\left(\frac{305}{3} \zeta_{3}+\frac{199}{80} \pi^{4}+\frac{853}{24} \pi^{2}+\frac{5753}{16}\right)\right]+\mathcal{O}\left(\varepsilon^{3}\right)\right\} \\
& +\left(\frac{\alpha_{s}}{\pi}\right)^{3} C_{F}\left\{-C_{F}^{2}\left(28 a_{4}+\frac{7}{6} a_{1}^{4}-\frac{3}{2} \pi^{2} a_{1}^{2}-\frac{223}{12} \pi^{2} a_{1}+\frac{5}{16} \zeta_{5}-\frac{\pi^{2}}{8} \zeta_{3}+\frac{157}{8} \zeta_{3}+\frac{19}{240} \pi^{4}+\frac{4801}{576} \pi^{2}+\frac{3023}{768}\right)\right. \\
& -C_{F} C_{A}\left(\frac{a_{4}}{6}+\frac{a_{1}^{4}}{144}+\frac{181}{72} \pi^{2} a_{1}^{2}+\frac{43}{9} \pi^{2} a_{1}-\frac{145}{16} \zeta_{5}+\frac{45}{16} \pi^{2} \zeta_{3}+\frac{289}{24} \zeta_{3}-\frac{6697}{17280} \pi^{4}-\frac{2137}{576} \pi^{2}-\frac{24131}{4608}\right) \\
& +\frac{C_{A}^{2}}{2}\left[\frac{1}{3}\left(\frac{85}{2} a_{4}+\frac{85}{48} a_{1}^{4}+\frac{127}{24} \pi^{2} a_{1}^{2}-\frac{325}{24} \pi^{2} a_{1}-37 \zeta_{5}+\frac{127}{12} \pi^{2} \zeta_{3}+\frac{5857}{96} \zeta_{3}-\frac{3419}{3840} \pi^{4}-\frac{4339}{576} \pi^{2}-\frac{1654711}{20736}\right)\right. \\
& \left.+\frac{\xi}{8}\left(\frac{7}{24} \zeta_{5}+\frac{\pi^{2}}{9} \zeta_{3}-\frac{13}{16} \zeta_{3}+\frac{17}{1728} \pi^{4}-\frac{\pi^{2}}{16}-\frac{13}{48}\right)\right] \\
& +C_{F} T_{F} n_{h}\left(12 a_{4}+\frac{a_{1}^{4}}{2}-\frac{\pi^{2}}{2} a_{1}^{2}+\frac{17}{9} \pi^{2} a_{1}+\frac{233}{288} \zeta_{3}+\frac{31}{720} \pi^{4}-\frac{553}{324} \pi^{2}-\frac{13571}{3456}\right) \\
& -C_{A} T_{F} n_{h}\left[\left(8 a_{4}+\frac{a_{1}^{4}}{3}-\frac{\pi^{2}}{3} a_{1}^{2}-\frac{80}{9} \pi^{2} a_{1}+\frac{15}{16} \zeta_{5}-\frac{11}{48} \pi^{2} \zeta_{3}+\frac{2813}{576} \zeta_{3}+\frac{17}{360} \pi^{4}+\frac{9067}{1296} \pi^{2}-\frac{788639}{41472}\right)\right. \\
& \left.+\frac{\xi}{24}\left(\zeta_{3}-\frac{2387}{576}\right)\right]
\end{aligned}
$$




$$
\begin{aligned}
& +\frac{C_{F} T_{F} n_{l}}{3}\left(16 a_{4}+\frac{2}{3} a_{1}^{4}+\frac{4}{3} \pi^{2} a_{1}^{2}-\frac{47}{6} \pi^{2} a_{1}+\frac{137}{8} \zeta_{3}-\frac{229}{720} \pi^{4}+\frac{113}{24} \pi^{2}+\frac{35}{6}\right) \\
& -\frac{C_{A} T_{F} n_{l}}{3}\left(8 a_{4}+\frac{a_{1}^{4}}{3}+\frac{2}{3} \pi^{2} a_{1}^{2}-\frac{47}{12} \pi^{2} a_{1}+\frac{35}{24} \zeta_{3}-\frac{19}{360} \pi^{4}-\frac{13}{16} \pi^{2}-\frac{111791}{5184}\right) \\
& +\frac{\left(T_{F} n_{h}\right)^{2}}{3}\left(7 \zeta_{3}+\frac{2}{15} \pi^{2}-\frac{8425}{864}\right)+\frac{T_{F}^{2} n_{h} n_{l}}{36}\left(13 \pi^{2}-\frac{4721}{36}\right)-\frac{\left(T_{F} n_{l}\right)^{2}}{18}\left(7 \zeta_{3}+\frac{19}{6} \pi^{2}+\frac{5767}{432}\right) \\
& +\varepsilon\left[-C_{F}^{2}\left(\frac{440}{3} a_{5}-16 \pi^{2} a_{4}+\frac{2444}{3} a_{4}-\frac{11}{9} a_{1}^{5}-\frac{2}{3} \pi^{2} a_{1}^{4}+\frac{611}{18} a_{1}^{4}+\frac{115}{27} \pi^{2} a_{1}^{3}+\frac{2}{3} \pi^{4} a_{1}^{2}+\frac{2309}{36} \pi^{2} a_{1}^{2}-14 \pi^{2} \zeta_{3} a_{1}\right.\right. \\
& \left.+\frac{751}{432} \pi^{4} a_{1}-\frac{367}{2} \pi^{2} a_{1}-\frac{53}{2} \zeta_{5}-\frac{29}{32} \zeta_{3}^{2}-\frac{5861}{288} \pi^{2} \zeta_{3}+\frac{5119}{16} \zeta_{3}+\frac{899}{5670} \pi^{6}-\frac{54467}{34560} \pi^{4}+\frac{74245}{2048} \pi^{2}+\frac{19337}{1536}\right) \\
& -C_{F} C_{A}\left(\frac{487}{3} a_{5}-6 \pi^{2} a_{4}-\frac{1796}{9} a_{4}-\frac{487}{360} a_{1}^{5}-\frac{\pi^{2}}{4} a_{1}^{4}-\frac{449}{54} a_{1}^{4}-\frac{1135}{108} \pi^{2} a_{1}^{3}+\frac{\pi^{4}}{4} a_{1}^{2}+\frac{7235}{216} \pi^{2} a_{1}^{2}-\frac{21}{4} \pi^{2} \zeta_{3} a_{1}\right. \\
& -\frac{949}{1080} \pi^{4} a_{1}+\frac{30803}{432} \pi^{2} a_{1}-\frac{125473}{384} \zeta_{5}+\frac{143}{4} \zeta_{3}^{2}+\frac{2703}{128} \pi^{2} \zeta_{3}-\frac{16339}{288} \zeta_{3}+\frac{27331}{181440} \pi^{6}-\frac{496741}{103680} \pi^{4}-\frac{17665}{55296} \pi^{2} \\
& \left.-\frac{861659}{27648}\right) \\
& +C_{A}^{2}\left[\frac{707}{6} a_{5}-7 \pi^{2} a_{4}+\frac{935}{9} a_{4}-\frac{707}{720} a_{1}^{5}-\frac{7}{24} \pi^{2} a_{1}^{4}+\frac{935}{216} a_{1}^{4}-\frac{905}{216} \pi^{2} a_{1}^{3}+\frac{7}{24} \pi^{4} a_{1}^{2}+\frac{7081}{216} \pi^{2} a_{1}^{2}-\frac{49}{8} \pi^{2} \zeta_{3} a_{1}\right. \\
& -\frac{41}{8640} \pi^{4} a_{1}-\frac{8833}{864} \pi^{2} a_{1}-\frac{41569}{256} \zeta_{5}+\frac{7451}{384} \zeta_{3}^{2}+\frac{14915}{4608} \pi^{2} \zeta_{3}+\frac{67807}{3456} \zeta_{3}+\frac{45047}{362880} \pi^{6}-\frac{126391}{51840} \pi^{4}-\frac{150229}{41472} \pi^{2} \\
& \left.-\frac{72476083}{746496}-\frac{\xi}{128}\left(\frac{149}{6} \zeta_{5}-\frac{25}{3} \zeta_{3}^{2}-\frac{77}{72} \pi^{2} \zeta_{3}+\frac{63}{2} \zeta_{3}-\frac{49}{405} \pi^{6}-\frac{383}{1080} \pi^{4}+\frac{35}{8} \pi^{2}+\frac{35}{2}\right)\right] \\
& +C_{F} T_{F} n_{h}\left(72 a_{5}-\frac{229}{6} a_{4}-\frac{3}{5} a_{1}^{5}-\frac{229}{144} a_{1}^{4}+\pi^{2} a_{1}^{3}-\frac{2219}{144} \pi^{2} a_{1}^{2}+\frac{143}{180} \pi^{4} a_{1}+\frac{293}{6} \pi^{2} a_{1}-\frac{87}{8} \zeta_{5}-\frac{81}{8} \pi^{2} \zeta_{3}\right. \\
& \left.-\frac{10913}{192} \zeta_{3}+\frac{3649}{8640} \pi^{4}-\frac{818609}{41472} \pi^{2}+\frac{164069}{6912}\right) \\
& -C_{A} T_{F} n_{h}\left[48 a_{5}-8 \pi^{2} a_{4}+\frac{4247}{12} a_{4}-\frac{2}{5} a_{1}^{5}-\frac{\pi^{2}}{3} a_{1}^{4}+\frac{4247}{288} a_{1}^{4}+\frac{2}{3} \pi^{2} a_{1}^{3}+\frac{\pi^{4}}{3} a_{1}^{2}+\frac{18133}{288} \pi^{2} a_{1}^{2}-7 \pi^{2} \zeta_{3} a_{1}\right. \\
& +\frac{97}{180} \pi^{4} a_{1}-\frac{775}{9} \pi^{2} a_{1}+\frac{551}{64} \zeta_{5}-\frac{181}{32} \zeta_{3}^{2}-\frac{549}{64} \pi^{2} \zeta_{3}+\frac{88855}{384} \zeta_{3}+\frac{1501}{15120} \pi^{6}-\frac{12607}{5760} \pi^{4}+\frac{286961}{13824} \pi^{2} \\
& \left.-\frac{35801821}{248832}-\frac{\xi}{8}\left(\zeta_{3}+\frac{\pi^{4}}{60}-\frac{121}{1728} \pi^{2}-\frac{7367}{1152}\right)\right] \\
& +\frac{C_{F} T_{F} n_{l}}{3}\left(224 a_{5}+\frac{1028}{3} a_{4}-\frac{28}{15} a_{1}^{5}+\frac{257}{18} a_{1}^{4}-\frac{56}{9} \pi^{2} a_{1}^{3}+\frac{257}{9} \pi^{2} a_{1}^{2}-\frac{17}{90} \pi^{4} a_{1}-\frac{539}{9} \pi^{2} a_{1}-\frac{1027}{4} \zeta_{5}\right. \\
& \left.-\frac{119}{16} \pi^{2} \zeta_{3}+\frac{1081}{6} \zeta_{3}-\frac{18599}{8640} \pi^{4}+\frac{160081}{4608} \pi^{2}+\frac{3103}{72}\right) \\
& -C_{A} T_{F} n_{l}\left(\frac{112}{3} a_{5}+\frac{514}{9} a_{4}-\frac{14}{45} a_{1}^{5}+\frac{257}{108} a_{1}^{4}-\frac{28}{27} \pi^{2} a_{1}^{3}+\frac{257}{54} \pi^{2} a_{1}^{2}-\frac{17}{540} \pi^{4} a_{1}-\frac{539}{54} \pi^{2} a_{1}-\frac{859}{24} \zeta_{5}\right. \\
& \left.-\frac{11}{16} \pi^{2} \zeta_{3}+\frac{1229}{432} \zeta_{3}-\frac{3691}{6480} \pi^{4}-\frac{1991}{648} \pi^{2}-\frac{4500377}{93312}\right) \\
& +\frac{\left(T_{F} n_{h}\right)^{2}}{3}\left(56 a_{4}+\frac{7}{3} a_{1}^{4}-\frac{7}{3} \pi^{2} a_{1}^{2}-\frac{4}{5} \pi^{2} a_{1}+\frac{3221}{80} \zeta_{3}-\frac{31}{72} \pi^{4}+\frac{39661}{7200} \pi^{2}-\frac{636911}{8640}\right) \\
& +T_{F}^{2} n_{h} n_{l}\left(\frac{32}{3} a_{4}+\frac{4}{9} a_{1}^{4}+\frac{8}{9} \pi^{2} a_{1}^{2}-\frac{35}{9} \pi^{2} a_{1}+\frac{27}{2} \zeta_{3}+\frac{179}{1080} \pi^{4}+\frac{2245}{1296} \pi^{2}-\frac{264817}{7776}\right)
\end{aligned}
$$




$$
\begin{aligned}
& \left.\left.-\frac{\left(T_{F} n_{l}\right)^{2}}{54}\left(275 \zeta_{3}+\frac{23}{5} \pi^{4}+\frac{1081}{16} \pi^{2}+\frac{253783}{864}\right)\right]+\mathcal{O}\left(\varepsilon^{2}\right)\right\} \\
& +\left(\frac{\alpha_{s}}{\pi}\right)^{4}\left\{C _ { F } ^ { 4 } \left[L_{0}-\frac{139}{2} a_{5}+12 \pi^{2} a_{4}-\frac{9137}{16} a_{4}+\frac{139}{240} a_{1}^{5}+\frac{\pi^{2}}{2} a_{1}^{4}-\frac{9137}{384} a_{1}^{4}-\frac{311}{72} \pi^{2} a_{1}^{3}-\frac{\pi^{4}}{2} a_{1}^{2}-\frac{8597}{192} \pi^{2} a_{1}^{2}\right.\right. \\
& +\frac{21}{2} \pi^{2} \zeta_{3} a_{1}-\frac{2783}{2880} \pi^{4} a_{1}+\frac{33687}{256} \pi^{2} a_{1}-\frac{2937}{128} \zeta_{5}+\frac{87}{128} \zeta_{3}^{2}+\frac{2755}{192} \pi^{2} \zeta_{3}-\frac{113181}{512} \zeta_{3}-\frac{899}{7560} \pi^{6}+\frac{18553}{23040} \pi^{4} \\
& \left.-\frac{24129}{1024} \pi^{2}-\frac{90577}{8192}\right] \\
& +C_{F}^{3} C_{A}(14.12 \pm 3.6)-C_{F}^{2} C_{A}^{2}[8.75607 \pm 2.9-(0.00269 \pm 0.0012) \xi] \\
& -C_{F} C_{A}^{3}\left[142.552 \pm 0.82-(0.43649 \pm 0.00076) \xi+(0.0205278 \pm 0.00012) \xi^{2}\right] \\
& +d_{F A}\left[9.4 \pm 2.1+(0.147 \pm 0.013) \xi-(0.0748 \pm 0.0028) \xi^{2}\right] \\
& +C_{F}^{3} T_{F} n_{h}\left[L_{1}+\frac{46}{3} a_{5}+16 \pi^{2} a_{4}-\frac{35189}{48} a_{4}-\frac{23}{180} a_{1}^{5}+\frac{2}{3} \pi^{2} a_{1}^{4}-\frac{35189}{1152} a_{1}^{4}-\frac{703}{108} \pi^{2} a_{1}^{3}-\frac{2}{3} \pi^{4} a_{1}^{2}-\frac{77155}{1152} \pi^{2} a_{1}^{2}\right. \\
& +14 \pi^{2} \zeta_{3} a_{1}-\frac{569}{2160} \pi^{4} a_{1}+\frac{3273}{16} \pi^{2} a_{1}-\frac{3067}{32} \zeta_{5}+\frac{29}{32} \zeta_{3}^{2}+\frac{2981}{288} \pi^{2} \zeta_{3}-\frac{119743}{384} \zeta_{3}-\frac{899}{5670} \pi^{6}+\frac{65953}{69120} \pi^{4} \\
& \left.-\frac{572525}{13824} \pi^{2}-\frac{305411}{36864}\right] \\
& +C_{F}^{2} C_{A} T_{F} n_{h}[14.893 \pm 0.083-(0.657352 \pm 0.00024) \xi] \\
& -C_{F} C_{A}^{2} T_{F} n_{h}\left[3.1601 \pm 0.056-(0.198984 \pm 0.00013) \xi+0.0244254 \xi^{2}\right] \\
& +C_{F}^{2}\left(T_{F} n_{h}\right)^{2}\left[L_{2}+120 a_{5}+\frac{2749}{48} a_{4}-a_{1}^{5}+\frac{2749}{1152} a_{1}^{4}-\frac{\pi^{2}}{3} a_{1}^{3}-\frac{10525}{1152} \pi^{2} a_{1}^{2}+\frac{43}{36} \pi^{4} a_{1}+\frac{711}{20} \pi^{2} a_{1}-\frac{493}{8} \zeta_{5}\right. \\
& \left.-\frac{269}{24} \pi^{2} \zeta_{3}-\frac{10127}{2560} \zeta_{3}-\frac{5513}{13824} \pi^{4}-\frac{678719}{64800} \pi^{2}-\frac{8452817}{414720}\right] \\
& -C_{F} C_{A}\left(T_{F} n_{h}\right)^{2}[0.01995 \pm 0.0062-0.10436 \xi] \\
& +C_{F}\left(T_{F} n_{h}\right)^{3}\left[L_{3}+\frac{1}{3}\left(104 a_{4}+\frac{13}{3} a_{1}^{4}+\frac{5}{3} \pi^{2} a_{1}^{2}-\frac{103}{10} \pi^{2} a_{1}+\frac{5881}{80} \zeta_{3}-\frac{299}{360} \pi^{4}+\frac{31451}{2700} \pi^{2}-\frac{5981281}{51840}\right)\right] \\
& +d_{F F} n_{h} L_{l}-C_{F}^{3} T_{F} n_{l}(4.92605 \pm 0.0067)+C_{F}^{2} C_{A} T_{F} n_{l}(15.0599 \pm 0.012) \\
& +C_{F} C_{A}^{2} T_{F} n_{l}[166.421 \pm 0.031-0.134051 \xi]-C_{F}^{2} T_{F}^{2} n_{h} n_{l}(5.08715 \pm 0.000074) \\
& +C_{F} C_{A} T_{F}^{2} n_{h} n_{l}[0.53235 \pm 0.0015+0.0910988 \xi]+0.0138079 C_{F} T_{F}^{3} n_{h}^{2} n_{l}-d_{F F} n_{l}(2.18 \pm 0.8) \\
& -C_{F}^{2}\left(T_{F} n_{l}\right)^{2}\left(\frac{32}{3} a_{5}+\frac{188}{9} a_{4}-\frac{4}{45} a_{1}^{5}+\frac{47}{54} a_{1}^{4}-\frac{8}{27} \pi^{2} a_{1}^{3}+\frac{47}{27} \pi^{2} a_{1}^{2}-\frac{31}{270} \pi^{4} a_{1}-\frac{239}{54} \pi^{2} a_{1}-\frac{601}{48} \zeta_{5}-\frac{\pi^{2}}{2} \zeta_{3}\right. \\
& \left.+\frac{6925}{576} \zeta_{3}-\frac{1181}{10368} \pi^{4}+\frac{1043}{384} \pi^{2}+\frac{3146969}{497664}\right) \\
& +\frac{C_{F} C_{A}\left(T_{F} n_{l}\right)^{2}}{3}\left(16 a_{5}+\frac{94}{3} a_{4}-\frac{2}{15} a_{1}^{5}+\frac{47}{36} a_{1}^{4}-\frac{4}{9} \pi^{2} a_{1}^{3}+\frac{47}{18} \pi^{2} a_{1}^{2}-\frac{31}{180} \pi^{4} a_{1}-\frac{239}{36} \pi^{2} a_{1}-\frac{365}{32} \zeta_{5}-\frac{11}{12} \pi^{2} \zeta_{3}\right. \\
& \left.-\frac{1111}{64} \zeta_{3}-\frac{4333}{17280} \pi^{4}-\frac{6815}{1152} \pi^{2}-\frac{4767085}{165888}\right) \\
& \left.+\frac{C_{F} T_{F}^{3} n_{h} n_{l}^{2}}{3}\left(\frac{\zeta_{3}}{16}-\frac{4}{15} \pi^{4}+\frac{19}{27} \pi^{2}+\frac{399325}{20736}\right)+\frac{C_{F}\left(T_{F} n_{l}\right)^{3}}{216}\left(\frac{467}{2} \zeta_{3}+\frac{71}{20} \pi^{4}+\frac{167}{3} \pi^{2}+\frac{103933}{864}\right)+\mathcal{O}(\varepsilon)\right\} \\
& +\mathcal{O}\left(\alpha_{s}^{5}\right)
\end{aligned}
$$


where $\alpha_{s}=\alpha_{s}^{\left(n_{f}\right)}(M), \xi=\xi^{\left(n_{f}\right)}(M) . L_{0, l, 1,2,3}$ are the $\varepsilon^{0}$ parts of the quantities $Z_{2}^{(4,0)}, Z_{2}^{(4, l)}, Z_{2}^{(4,1)}, Z_{2}^{(4,2)}, Z_{2}^{(4,3)}$ given in Eqs. (28-32) of [14]. Their numerical values are given in Eqs. (5-9) of that paper. The finite four-loop terms of Eq. (7) are equal to the corresponding finite four-loop terms in $Z_{Q}^{\text {os }}$ plus products of lower-loop quantities which are all known analytically. For 14 out of 23 color structures these coefficients in $Z_{Q}^{\text {os }}$ are only known numerically [13]. We use these numerical values, together with their uncertainty estimates, from the Tables V, VI, and VII of that paper. Note that in Ref. [13] $Z_{Q}^{\text {os }}$ has been computed in an expansion in $\xi$ up to the second order; 9 out of these 19 color structures are obviously gauge invariant, and 7 more seem to be either gauge-invariant or have at most linear $\xi$ terms (though we know no explicit proof). The remaining 3 structures $\left(C_{F} C_{A}^{3}, d_{F A}, C_{F} C_{A}^{2} T_{F} n_{h}\right)$ may contain terms with higher powers of $\xi$, which are not known. The same is true for the corresponding terms in $z(M)$ in Eq. (7).

If we re-express $z(M)$ in Eq. (7) via $\alpha_{s}^{\left(n_{l}\right)}(M)$, the terms up to three loops agree with [5]. (Note that positive powers of $\varepsilon$ are not presented [5].) The $\alpha_{s}^{4} n_{l}^{3}$ term also agrees with [5].

After specifying the color factors to QCD with $N_{c}=3$ we obtain for $\varepsilon=0$

$$
\begin{aligned}
z(M)= & 1-\frac{4}{3} \frac{\alpha_{s}}{\pi}-\left(\frac{\alpha_{s}}{\pi}\right)^{2}\left(17.45-1.33 n_{l}\right) \\
& -\left(\frac{\alpha_{s}}{\pi}\right)^{3}\left(262.42-0.78 \xi-35.81 n_{l}+0.98 n_{l}^{2}\right) \\
& -\left(\frac{\alpha_{s}}{\pi}\right)^{4}\left[5137.52-15.67 \xi+1.07 \xi^{2}\right. \\
& \left.-(1030.82-0.71 \xi) n_{l}+60.30 n_{l}^{2}-1.00 n_{l}^{3}\right] \\
& +\mathcal{O}\left(\alpha_{s}^{5}\right) .
\end{aligned}
$$

In Landau gauge $\left(\xi^{\left(n_{f}\right)}=1\right)$ at $n_{l}=4$ this gives

$$
\begin{aligned}
z(M)= & 1-\frac{4}{3} \frac{\alpha_{s}}{\pi}-12.12\left(\frac{\alpha_{s}}{\pi}\right)^{2}-134.11\left(\frac{\alpha_{s}}{\pi}\right)^{3} \\
& -1903.22\left(\frac{\alpha_{s}}{\pi}\right)^{4}+\mathcal{O}\left(\alpha_{s}^{5}\right),
\end{aligned}
$$

while the naive non-Abelianization [22] (large $\beta_{0}$ limit) predicts [5]

$$
\begin{aligned}
1 & -\frac{4}{3} \frac{\alpha_{s}}{\pi}-16.66\left(\frac{\alpha_{s}}{\pi}\right)^{2}-153.41\left(\frac{\alpha_{s}}{\pi}\right)^{3} \\
& -1953.40\left(\frac{\alpha_{s}}{\pi}\right)^{4}+\mathcal{O}\left(\alpha_{s}^{5}\right) .
\end{aligned}
$$

The comparison to Eq. (9) shows that up to four loops these predictions are rather good. The coefficients are all negative and grow very fast, which can be explained by the infrared renormalon at $u=1 / 2$ [5]. This is the closest possible position of a renormalon singularity in the Borel plane $u$ to the origin, and it leads to the fastest possible growth of perturbative terms $(L-1) !\left(\beta_{0} / 2\right)^{L}\left(\alpha_{s} / \pi\right)^{L}$. The coefficients of powers of $\xi$ are much smaller than the $\xi$-independent terms.

\section{EFFECT OF A LIGHTER-FLAVOR MASS}

Now we suppose that $n_{m}$ light flavors have a nonzero mass $m$, while the remaining $n_{0}=n_{l}-n_{m}$ light flavors are massless. In practice, $n_{m}=1$, e.g., $c$ in $b$-quark HQET. In this case the massless result (7) for the matching coefficient should be multiplied by the additional factor

$$
z^{\prime}=\frac{Z_{Q}^{\mathrm{os}}\left(g_{0}^{\left(n_{f}\right)}, \xi_{0}^{\left(n_{f}\right)}, m_{0}^{\left(n_{f}\right)}\right)}{Z_{Q}^{\mathrm{os}}\left(g_{0}^{\left(n_{f}\right)}, \xi_{0}^{\left(n_{f}\right)}, 0\right)} \frac{Z_{h}^{\mathrm{os}}\left(g_{0}^{\left(n_{l}\right)}, \xi_{0}^{\left(n_{l}\right)}, 0\right)}{Z_{h}^{\mathrm{os}}\left(g_{0}^{\left(n_{l}\right)}, \xi_{0}^{\left(n_{l}\right)}, m_{0}^{\left(n_{l}\right)}\right)},
$$

where $\quad Z_{Q, h}^{\text {os }}(\ldots, 0) \equiv Z_{Q, h}^{\text {os }}(\ldots) \quad$ in $\quad$ Eq. $\quad$ (2) and $Z_{h}^{\text {os }}\left(g_{0}^{\left(n_{l}\right)}, \xi_{0}^{\left(n_{l}\right)}, 0\right)=1$. This factor does not depend on the renormalization scale $\mu$. In the expression

$$
\begin{aligned}
\log z^{\prime}= & \log Z_{Q}^{\text {os }}\left(g_{0}^{\left(n_{f}\right)}, \xi_{0}^{\left(n_{f}\right)}, m_{0}^{\left(n_{f}\right)}\right) \\
& -\log Z_{Q}^{\text {os }}\left(g_{0}^{\left(n_{f}\right)}, \xi_{0}^{\left(n_{f}\right)}, 0\right) \\
& -\log Z_{h}^{\text {os }}\left(g_{0}^{\left(n_{l}\right)}, \xi_{0}^{\left(n_{l}\right)}, m_{0}^{\left(n_{l}\right)}\right)
\end{aligned}
$$

we reexpress all terms via $\alpha_{s}^{\left(n_{f}\right)}(M), \xi^{\left(n_{f}\right)}(M)$ and the on-shell lighter-flavor mass $m$ (it is the same in both $n_{f}$ and $n_{l}$ flavor theories). The result depends on the dimensionless ratio

$$
x=\frac{m}{M} .
$$

If we express $z^{\prime}$ via $\alpha_{s}^{\left(n_{f}\right)}(\mu), \xi^{\left(n_{f}\right)}(\mu)$, the coefficients will depend on $\mu$. This dependence is determined by the renormalization group equation

$$
\frac{d \log z^{\prime}}{d \log \mu}=0
$$

together with

$$
\begin{gathered}
\frac{d \log \alpha_{s}^{\left(n_{f}\right)}(\mu)}{d \log \mu}=-2 \varepsilon-2 \beta^{\left(n_{f}\right)}\left(\alpha_{s}^{\left(n_{f}\right)}(\mu)\right), \\
\frac{d \log \left(1-\xi^{\left(n_{f}\right)}(\mu)\right)}{d \log \mu}=-\gamma_{A}^{\left(n_{f}\right)}\left(\alpha_{s}^{\left(n_{f}\right)}(\mu), \xi^{\left(n_{f}\right)}(\mu)\right) .
\end{gathered}
$$

Ultraviolet divergences cancel in each fraction in (11). On the other hand, the on-shell wave function renormalization factors have extra infrared divergences at $m=0$. 


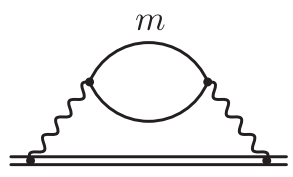

(a)

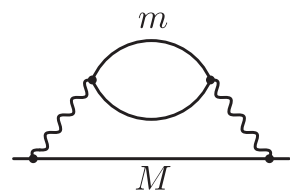

(b)
FIG. 1. Two-loop contributions to the on-shell wave-function renormalization constants: (a) in HQET; (b) in QCD.

However, $z^{\prime}$ in Eq. (11) has a smooth limit for $x \rightarrow 0$. In the following we illustrate the cancellation for infrared divergences at two-loop order. Similar mechanisms are also at work at higher loop orders. For dimensional reasons the two-loop corrections in Fig. 1(a) lead to $\log Z_{h}^{\text {os }}(m) \sim$ $g_{0}^{4} m^{-4 \varepsilon}$. Furthermore, we have $\log Z_{h}^{\text {os }}(0)=0$. Thus, the limit $x \rightarrow 0$ is discontinuous. In QCD [Fig. 1(b)] we have $\log Z_{Q}^{\text {os }}(0) \sim g_{0}^{4} M^{-4 \varepsilon}$ for dimensional reasons. For $m \ll M$ there are 3 regions (see $[28,29]$ ):

(i) Hard (all momenta $\sim M$ ): a regular series in $m^{2},\left.\log Z_{Q}^{\text {os }}(m)\right|_{\text {hard }}=\log Z_{Q}^{\text {os }}(0)\left[1+\mathcal{O}\left(x^{2}\right)\right]$.

(ii) Soft-hard (momentum of one $m$-line is $\sim m$, all the remaining momenta are $\sim M$ ). If we take the term $m$ from the numerator $\not k+m$ of the soft propagator, there is another factor $m$ in the numerator of the hard mass- $m$ propagator, and the soft-loop integral is $\sim m^{2-2 \varepsilon}$; if we take $\not k$ instead, we have to expand the hard subdiagram in $k$ up to the linear term, and the soft loop is $\sim m^{4-2 \varepsilon}$. We obtain $\left.\log Z_{Q}^{\text {os }}(m)\right|_{\text {soft-hard }} \sim$ $g_{0}^{4} M^{-2 \varepsilon} m^{-2 \varepsilon} x^{4}$.

(iii) Soft (all momenta $\sim m$ ): the leading term is the HQET one, the Taylor series is in $x$ (not in $x^{2}$ ), $\left.\log Z_{Q}^{\text {os }}(m)\right|_{\text {soft }}=\log Z_{h}^{\text {os }}(m)[1+\mathcal{O}(x)]$.
As a result, $\left.\log Z_{Q}^{\text {os }}(m)\right|_{\text {hard }}-\log Z_{Q}^{\text {os }}(0)$ is smooth at $x \rightarrow 0$; $\left.\log Z_{Q}^{\text {os }}(m)\right|_{\text {soft-hard }}$ is subleading and hence smooth; $\left.\log Z_{Q}^{\text {os }}(m)\right|_{\text {soft }}$ has the same discontinuity as $\log Z_{h}^{\text {os }}$; hence $\log z^{\prime}(12)$ has a smooth limit 1 at $x \rightarrow 0$.

The two-loop term in $Z_{Q}^{\text {os }}\left(g_{0}^{\left(n_{f}\right)}, \xi_{0}^{\left(n_{f}\right)}, m_{0}^{\left(n_{f}\right)}\right)$ has been calculated up to $\varepsilon^{0}$ in [7]; the result exact in $\varepsilon$ has been obtained in [30]. The three-loop term has been calculated up to $\varepsilon^{0}$ in [31]. Some master integrals are only known as truncated series in $x$ or as numerical interpolations, see [32] for detailed discussion of these master integrals. Exact results in $x$ for the coefficient of $C_{F} T_{F}^{2} n_{m} n_{0} \alpha_{s}^{3}$ can be obtained using the formulas of Appendix B.

The HQET renormalization constant $Z_{h}^{\text {os }}\left(g_{0}^{\left(n_{l}\right)}, \xi_{0}^{\left(n_{l}\right)}, m_{0}^{\left(n_{l}\right)}\right)$ at two loops has been calculated in [22], and at three loops in [33] (one of the master integrals is discussed in [34]; note that there are some typos in formulas in the journal version of [33] fixed later in arXiv).

Altogether we are now in the position to obtain $z^{\prime}$ up to three loops. The expansion of $z^{\prime}$ in terms of $\alpha_{s}^{\left(n_{f}\right)}(M)$ and its decomposition into color factors is given by

$$
\begin{aligned}
z^{\prime}= & 1+C_{F} T_{F}\left(\frac{\alpha_{s}^{\left(n_{f}\right)}(M)}{\pi}\right)^{2}\left(A_{0}+A_{1} \varepsilon+\mathcal{O}\left(\varepsilon^{2}\right)\right) \\
& +C_{F} T_{F}\left(\frac{\alpha_{s}^{\left(n_{f}\right)}(M)}{\pi}\right)^{3}\left(C_{F} A_{F}+C_{A} A_{A}+T_{F} n_{0} A_{l}\right. \\
& \left.+T_{F} n_{m} A_{m}+T_{F} n_{h} A_{h}+\mathcal{O}(\varepsilon)\right)+\mathcal{O}\left(\alpha_{s}^{4}\right),
\end{aligned}
$$

where

$$
\begin{aligned}
A_{0}= & \frac{1}{4}\left[(1-x)\left(2-x-x^{2}-6 x^{3}\right) H_{1,0}(x)-(1+x)\left(2+x-x^{2}+6 x^{3}\right) H_{-1,0}(x)\right. \\
& \left.-\frac{3}{2} \pi^{2} x+(4 \log x+7) x^{2}-\frac{5}{2} \pi^{2} x^{3}+\left(6 \log ^{2} x+\pi^{2}\right) x^{4}\right] .
\end{aligned}
$$

The expansion of this function in $x$ reads

$$
\begin{aligned}
A_{0} & =\frac{1}{4}\left[-\frac{3}{2} \pi^{2} x+12 x^{2}-\frac{5}{2} \pi^{2} x^{3}+\left(6 \log ^{2} x-11 \log x+\pi^{2}+\frac{125}{12}\right) x^{4}+\sum_{n=3}^{\infty}\left(2 g(2 n) \log x+\frac{d g(2 n)}{d n}\right) x^{2 n}\right], \\
g(x) & =\frac{2}{x}-\frac{3}{x-1}-\frac{5}{x-3}+\frac{6}{x-4} .
\end{aligned}
$$

Note that the only terms with odd powers of $x$ are $x^{1}$ and $x^{3}$. The expansion in $x^{-1}$ is given by

$$
A_{0}=\frac{1}{4}\left[-2 \log ^{2} x^{-1}+\frac{19}{3} \log x^{-1}-\frac{\pi^{2}}{3}-\frac{229}{36}+\sum_{n=1}^{\infty}\left(2 g(-2 n) \log x^{-1}+\frac{d g(-2 n)}{d n}\right) x^{-2 n}\right] .
$$

For illustration we show in Fig. $2 A_{0}(x)$ for $x \in[0,1]$. The $\mathcal{O}(\varepsilon)$ term at two loops reads 


$$
\begin{aligned}
A_{1}= & \frac{1}{4}\left[(1-x)\left(2-x-x^{2}-6 x^{3}\right)\left(2 H_{1,1,0}(x)-4 H_{1,-1,0}(x)\right)\right. \\
& +(1+x)\left(2+x-x^{2}+6 x^{3}\right)\left(2 H_{-1,-1,0}(x)-4 H_{-1,1,0}(x)-\pi^{2} H_{-1}(x)\right) \\
& +(1-x)\left(9-6 x+6 x^{2}-17 x^{3}\right) H_{1,0}(x)-(1+x)\left(9+6 x+6 x^{2}+17 x^{3}\right) H_{-1,0}(x) \\
& +4 x\left(3+5 x^{2}\right)\left(H_{0,1,0}(x)+H_{0,-1,0}(x)\right)+6 \pi^{2}\left(L+2 a_{1}-\frac{5}{4}\right) x+\left(L+2 \pi^{2}+\frac{53}{2}\right) x^{2} \\
& \left.+10 \pi^{2}\left(L+2 a_{1}-\frac{23}{20}\right) x^{3}-12\left(L^{3}-\frac{17}{12} L^{2}-\zeta_{3}-\frac{17}{72} \pi^{2}\right) x^{4}\right] \\
= & \pi^{2}\left(\frac{3}{2} L+3 a_{1}-\frac{19}{8}\right) x+\frac{5}{2} x^{2}+\pi^{2}\left(\frac{5}{2} L+5 a_{1}-\frac{8}{3}\right) x^{3}-\left(3 L^{3}-\frac{17}{4} L^{2}-\frac{3}{8} L-3 \zeta_{3}+\frac{2}{3} \pi^{2}+\frac{2827}{288}\right) x^{4} \\
& -\frac{63}{80} \pi^{2} x^{5}-\frac{2}{15}\left(\frac{61}{5} L-2 \pi^{2}-\frac{4243}{225}\right) x^{6}-\frac{15}{112} \pi^{2} x^{7}-\frac{3}{56}\left(\frac{53}{35} L-\frac{3}{2} \pi^{2}-\frac{5909}{1960}\right) x^{8}+\mathcal{O}\left(x^{9}\right),
\end{aligned}
$$

where $L=\log x$.

At three-loop order the $C_{F} T_{F}^{2} n_{m} n_{0} \alpha_{s}^{3}$ term is known exactly via harmonic polylogarithms of $x$ :

$$
\begin{aligned}
A_{l}= & \frac{1}{3}\left[(1-x)\left(2-x-x^{2}-6 x^{3}\right)\left(H_{1,-1,0}(x)+\frac{\pi^{2}}{12} H_{1}(x)\right)+(1+x)\left(2+x-x^{2}+6 x^{3}\right)\left(H_{-1,1,0}(x)+\frac{5}{12} \pi^{2} H_{-1}(x)\right)\right. \\
& -\frac{1}{6}(1-x)\left(19-11 x+x^{2}-39 x^{3}\right) H_{1,0}(x)+\frac{1}{6}(1+x)\left(19+11 x+x^{2}+39 x^{3}\right) H_{-1,0}(x) \\
& -x\left(3+5 x^{2}\right)\left(H_{0,1,0}(x)+H_{0,-1,0}(x)\right)-\pi^{2}\left(\frac{3}{2} L+3 a_{1}-\frac{5}{2}\right) x-\left(\frac{17}{2} L+2 \pi^{2}+\frac{91}{4}\right) \frac{x^{2}}{3}-5 \pi^{2}\left(\frac{L}{2}+a_{1}-\frac{2}{3}\right) x^{3} \\
& \left.+\left(2 L^{3}-\frac{13}{2} L^{2}-\pi^{2} L-9 \zeta_{3}-\frac{13}{12} \pi^{2}\right) x^{4}\right] \\
= & -\pi^{2}\left(\frac{L}{2}+a_{1}-\frac{7}{6}\right) x-\frac{7}{3} x^{2}-\frac{5}{3} \pi^{2}\left(\frac{L}{2}+a_{1}-\frac{7}{12}\right) x^{3}+\left[\frac{2}{3} L^{3}-\frac{13}{6} L^{2}-\left(\frac{\pi^{2}}{3}-\frac{3}{4}\right) L-3 \zeta_{3}+\frac{\pi^{2}}{4}+\frac{1175}{432}\right] x^{4} \\
& +\frac{21}{40} \pi^{2} x^{5}+\frac{4}{45}\left(\frac{13}{5} L-\frac{4}{3} \pi^{2}-\frac{2414}{225}\right) x^{6}+\frac{5}{56} \pi^{2} x^{7}+\left(\frac{4}{35} L-\frac{\pi^{2}}{4}-\frac{40489}{29400}\right) \frac{x^{8}}{7}+\mathcal{O}\left(x^{9}\right),
\end{aligned}
$$

where after the second equality sign we show the expansion in $x$. In principle, it is straightforward to obtain exact results in $x$ also the four-loop $C_{F} T_{F}^{3} n_{m} n_{0}^{2} \alpha_{s}^{4}$ term. However, we refrain from presenting such results because the remaining four-loop color structures are not known.

The remaining three-loop terms can be obtained in a series expansion in $x$ with the help of the result from [31]. Including terms up to order $x^{8}$ gives

$$
\begin{aligned}
A_{F}= & \frac{\pi^{2}}{3}\left(8 a_{1}+\frac{13}{4} \pi-\frac{343}{24}\right) x-\left(L^{2}-\frac{67}{6} L-\frac{17}{8} \pi^{2}+\frac{229}{18}\right) x^{2}+\frac{\pi^{2}}{3}\left(\frac{11}{3} L+\frac{44}{3} a_{1}+\frac{35}{8} \pi-\frac{157}{8}\right) x^{3} \\
& +\left[\frac{19}{6} L^{3}-\frac{911}{120} L^{2}-\left(3 \pi^{2} a_{1}-\frac{3}{2} \zeta_{3}-\frac{45}{16} \pi^{2}-\frac{40567}{3600}\right) L+20 a_{4}+\frac{5}{6} a_{1}^{4}+\frac{2}{3} \pi^{2} a_{1}^{2}+\frac{11}{16} \pi^{2} a_{1}+\frac{387}{32} \zeta_{3}\right. \\
& \left.-\frac{43}{144} \pi^{4}-\frac{155}{64} \pi^{2}-\frac{2534579}{216000}\right] x^{4}+\frac{7}{5} \pi^{2}\left(\frac{3}{32} \pi+\frac{1}{5}\right) x^{5} \\
& +\left[\frac{1579}{70} L^{2}+\left(\frac{77}{16} \pi^{2}-\frac{328067}{11025}\right) L-\frac{1}{16}\left(77 \pi^{2} a_{1}-\frac{539}{2} \zeta_{3}-\frac{83}{15} \pi^{2}-\frac{126231437}{1157625}\right)\right] \frac{x^{6}}{9}-\frac{\pi^{2}}{28}\left(\frac{25}{16} \pi+\frac{1}{7}\right) x^{7} \\
& +\left[\frac{2843}{105} L^{2}+\left(\frac{21}{2} \pi^{2}-\frac{718639}{33075}\right) \frac{L}{2}-\frac{1}{4}\left(21 \pi^{2} a_{1}-\frac{147}{2} \zeta_{3}-\frac{4379}{240} \pi^{2}+\frac{1213332979}{83349000}\right)\right] \frac{x^{8}}{32}+\mathcal{O}\left(x^{9}\right),
\end{aligned}
$$




$$
\begin{aligned}
& A_{A}=\frac{\pi^{2}}{8}\left(\frac{25}{2} L+\frac{313}{3} a_{1}-\frac{13}{3} \pi-\frac{2473}{36}\right) x \\
& +\left[\frac{L^{2}}{2}+\left(\frac{3}{2} \zeta_{3}-\frac{31}{90} \pi^{4}+7 \pi^{2}-\frac{7}{3}\right) L-5 \zeta_{5}-\frac{7}{2} \pi^{2} \zeta_{3}+\frac{79}{4} \zeta_{3}-\frac{17}{180} \pi^{4}+\frac{35}{18} \pi^{2}+\frac{517}{9}\right] \frac{x^{2}}{4} \\
& +\frac{\pi^{2}}{24}\left(\frac{269}{6} L+\frac{1291}{3} a_{1}-\frac{35}{2} \pi-\frac{865}{3}\right) x^{3}-\left[\frac{83}{48} L^{3}+\left(3 \pi^{2}-\frac{3977}{60}\right) \frac{L^{2}}{8}-\left(\frac{3}{2} \pi^{2} a_{1}-3 \zeta_{3}-\frac{13}{24} \pi^{2}-\frac{230293}{28800}\right) L\right. \\
& \left.+10 a_{4}+\frac{5}{12} a_{1}^{4}+\frac{\pi^{2}}{3} a_{1}^{2}+\frac{11}{32} \pi^{2} a_{1}+\frac{111}{64} \zeta_{3}-\frac{161}{1440} \pi^{4}-\frac{631}{1152} \pi^{2}-\frac{452033}{864000}\right] x^{4}+\frac{\pi^{2}}{20}\left(\frac{79}{9} L-\frac{21}{16} \pi-\frac{2671}{432}\right) x^{5} \\
& +\left[\frac{5}{3} L^{3}+\frac{9911}{840} L^{2}-\left(\pi^{2}+\frac{8394157}{529200}\right) L+\frac{1}{12}\left(77 \pi^{2} a_{1}-\frac{509}{2} \zeta_{3}-\frac{3607}{60} \pi^{2}+\frac{8471770063}{18522000}\right)\right] \frac{x^{6}}{24} \\
& +\frac{\pi^{2}}{28}\left(\frac{57}{25} L+\frac{25}{32} \pi-\frac{11549}{14000}\right) x^{7}+\left[\frac{43}{27} L^{3}+\frac{209}{20} L^{2}+\left(125 \pi^{2}-\frac{12327647}{14700}\right) \frac{L}{216}\right. \\
& \left.+\frac{1}{8}\left(21 \pi^{2} a_{1}-\frac{1435}{18} \zeta_{3}-\frac{1213519}{45360} \pi^{2}+\frac{103012097}{2058000}\right)\right] \frac{x^{8}}{32}+\mathcal{O}\left(x^{9}\right), \\
& A_{h}=-\left(2 L+\frac{13}{5}\right) \frac{x^{2}}{5}+\frac{2}{15} \pi^{2} x^{3}+\left[\frac{3}{70} L^{2}+\left(\pi^{2}-\frac{35887}{4900}\right) \frac{L}{3}-\frac{1}{36}\left(13 \pi^{2}-\frac{59985349}{514500}\right)\right] x^{4} \\
& -\left(244 L^{2}-\frac{92779}{315} L+\frac{353877541}{793800}\right) \frac{x^{6}}{945}-\left(47 L^{2}+\frac{925823}{13860} L-\frac{4543985839}{384199200}\right) \frac{x^{8}}{770}+\mathcal{O}\left(x^{9}\right) \text {, } \\
& A_{m}=-\pi^{2}\left(\frac{L}{2}-\frac{2}{15}\right) x-\frac{7}{3} x^{2}-\frac{5}{6} \pi^{2} L x^{3}+\left[\frac{2}{3} L^{3}-\frac{13}{6} L^{2}-\left(\frac{\pi^{2}}{3}-\frac{15}{4}\right) L+\frac{1}{4}\left(\pi^{2}+\frac{203}{108}\right)\right] x^{4} \\
& -\left(\frac{308}{5} L+\frac{16}{3} \pi^{2}-\frac{13159}{225}\right) \frac{x^{6}}{45}+\left(3 L^{2}-\frac{751}{70} L+\frac{2095}{336}\right) \frac{x^{8}}{14}+\mathcal{O}\left(x^{9}\right) \text {. }
\end{aligned}
$$

Starting from three loops the individual terms in Eq. (12) are gauge parameter dependent. However, $\xi$ cancels in the three-loop expression for $z^{\prime}$. It might be that $z^{\prime}$ is gauge invariant to all orders, but we have no proof of this conjecture.

\section{THE QED AND BLOCH-NORDSIECK HEAVY-LEPTON FIELDS}

In QED the matching coefficient $z(\mu)$ is gauge invariant to all orders in $\alpha$ [5]. The proof given in this paper is literally valid only for $n_{f}=1$ lepton flavor, but can be easily generalized for any $n_{f}$, as we demonstrate in the following.

The QED on-shell renormalization constant $Z_{\psi}^{\text {os }}$ is gauge invariant to all orders $[10,35,36]$. Gauge dependence of the $\overline{\mathrm{MS}} Z_{\psi}$ can be found using the so-called LKF transformation $[37,38]$ for arbitrary $n_{f}$. In the gauge where the free photon propagator is

$$
D_{\mu \nu}^{0}(k)=\frac{1}{k^{2}}\left(g_{\mu \nu}-\frac{k_{\mu} k_{\nu}}{k^{2}}\right)+\Delta(k) k_{\mu} k_{\nu},
$$

the full bare lepton propagator reads

$$
\begin{aligned}
S(x) & =S_{L}(x) e^{-i e_{0}^{2}(\tilde{\Delta}(x)-\tilde{\Delta}(0))}, \\
\tilde{\Delta}(x) & =\int \frac{d^{d} k}{(2 \pi)^{d}} \Delta(k) e^{-i k x},
\end{aligned}
$$

where $S_{L}(x)$ is the Landau-gauge propagator. In the covariant gauge $\Delta(k)=\left(1-\xi_{0}\right) /\left(k^{2}\right)^{2}$, and $\tilde{\Delta}(0)=0$ in dimensional regularization. The lepton fields renormalization does not depend on their masses, so, let us assume that all $n_{f}$ flavors are massless. The propagator has a single Dirac structure

$$
S(x)=S_{0}(x) e^{\sigma(x)}
$$

where $S_{0}(x)$ is the $d$-dimensional free propagator. Then

$$
\sigma(x)=\sigma_{L}(x)+\left(1-\xi_{0}\right) \frac{e_{0}^{2}}{(4 \pi)^{d / 2}}\left(-\frac{x^{2}}{4}\right)^{\varepsilon} \Gamma(-\varepsilon)
$$

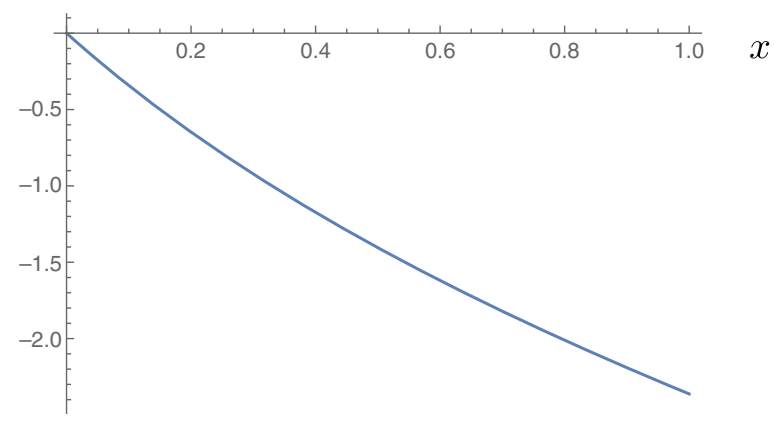

FIG. 2. The function $A_{0}(x)$. 
reexpressing this result via the renormalized quantities, we obtain

$$
\log Z_{\psi}(\alpha, \xi)=\log Z_{L}(\alpha)-(1-\xi) \frac{\alpha}{4 \pi \varepsilon} .
$$

In QED $Z_{A} Z_{\alpha}=1$ due to Ward identities, hence

$$
\frac{d \log ((1-\xi(\mu)) \alpha(\mu))}{d \log \mu}=-2 \varepsilon
$$

exactly, and the anomalous dimension

$$
\gamma_{\psi}(\alpha, \xi)=\gamma_{L}(\alpha)+2(1-\xi) \frac{\alpha}{4 \pi}
$$

contains $\xi$ only in the one-loop term.

In the Bloch-Nordsieck EFT with $n_{l}$ light lepton flavors $Z_{h}^{o s}$ is gauge-invariant (even if some of these flavors have nonzero masses). Gauge dependence of the $\overline{\mathrm{MS}} Z_{h}$ can be found using exponentiation. The full bare propagator is

$$
S_{h}(t)=S_{h 0}(t) \exp \left(\sum_{i} w_{i}\right)
$$

where $w_{i}$ are webs $[39,40]$. In QED all webs have even numbers of photon legs; all webs with $>2$ legs are gauge invariant; all 2-leg webs except the trivial one (the free photon propagator) are gauge invariant, too. Therefore,

$$
\log \frac{S_{h}(t)}{S_{h L}(t)}=\left(1-\xi_{0}\right) \frac{e_{0}^{2}}{(4 \pi)^{d / 2}}\left(\frac{i t}{2}\right)^{2 \varepsilon} \Gamma(-\varepsilon)
$$

reexpressing this result via the renormalized quantities, we obtain

$$
\begin{aligned}
\log Z_{h}(\alpha, \xi) & =\log Z_{h L}(\alpha)-(1-\xi) \frac{\alpha}{4 \pi \varepsilon}, \\
\gamma_{h}(\alpha, \xi) & =\gamma_{h L}(\alpha)+2(1-\xi) \frac{\alpha}{4 \pi}
\end{aligned}
$$

Finally, in the abelian case $\zeta_{\alpha}(\mu)=\zeta_{A}(\mu)^{-1}$ due to Ward identities, hence $\left.\left(1-\xi^{\left(n_{f}\right)}(\mu)\right) \alpha^{\left(n_{f}\right)}(\mu)\right)=$ $\left.\left(1-\xi^{\left(n_{l}\right)}(\mu)\right) \alpha^{\left(n_{l}\right)}(\mu)\right)$, and we arrive at the conclusion that $z(\mu)$ is gauge invariant (some light flavors may be massive, this does not matter).

Let us in the following specify $z(M)$ from Eq. (7) to QED. Setting $C_{F}=T_{F}=d_{F F}=1$ and $C_{A}=d_{F A}=0$ we see that our four-loop result is indeed gauge invariant and is given by

$$
\begin{aligned}
& z(M)=1-\frac{\alpha}{\pi}\left[1+\varepsilon\left(\frac{\pi^{2}}{16}+2\right)-\varepsilon^{2}\left(\frac{\zeta_{3}}{4}-\frac{\pi^{2}}{12}-4\right)-\varepsilon^{3}\left(\frac{\zeta_{3}}{3}-\frac{3}{640} \pi^{4}-\frac{\pi^{2}}{6}-8\right)+\mathcal{O}\left(\varepsilon^{4}\right)\right] \\
& +\left(\frac{\alpha}{\pi}\right)^{2}\left\{\pi^{2} a_{1}-\frac{3}{2} \zeta_{3}-\frac{55}{48} \pi^{2}+\frac{5957}{1152}+\frac{n_{l}}{12}\left(\pi^{2}+\frac{113}{8}\right)\right. \\
& +\varepsilon\left[-24 a_{4}-a_{1}^{4}-2 \pi^{2} a_{1}^{2}+\frac{31}{4} \pi^{2} a_{1}-\frac{203}{8} \zeta_{3}+\frac{7}{20} \pi^{4}-\frac{4903}{1152} \pi^{2}+\frac{56845}{6912}+n_{l}\left(\zeta_{3}+\frac{127}{288} \pi^{2}+\frac{851}{192}\right)\right] \\
& +\varepsilon^{2}\left[-144 a_{5}-186 a_{4}+\frac{6}{5} a_{1}^{5}-\frac{31}{4} a_{1}^{4}+4 \pi^{2} a_{1}^{3}-\frac{31}{2} \pi^{2} a_{1}^{2}-\frac{13}{15} \pi^{4} a_{1}+30 \pi^{2} a_{1}+\frac{609}{4} \zeta_{5}+\frac{11}{4} \pi^{2} \zeta_{3}-\frac{28169}{288} \zeta_{3}\right. \\
& \left.\left.+\frac{10007}{7680} \pi^{4}-\frac{114943}{6912} \pi^{2}+\frac{1838165}{41472}+\frac{n_{l}}{24}\left(\frac{305}{3} \zeta_{3}+\frac{199}{80} \pi^{4}+\frac{853}{24} \pi^{2}+\frac{5753}{16}\right)\right]+\mathcal{O}\left(\varepsilon^{3}\right)\right\} \\
& +\left(\frac{\alpha}{\pi}\right)^{3}\left\{-16 a_{4}-\frac{2}{3} a_{1}^{4}+\pi^{2} a_{1}^{2}+\frac{737}{36} \pi^{2} a_{1}-\frac{5}{16} \zeta_{5}+\frac{\pi^{2}}{8} \zeta_{3}-\frac{4747}{288} \zeta_{3}-\frac{13}{360} \pi^{4}-\frac{259133}{25920} \pi^{2}-\frac{230447}{20736}\right. \\
& +\frac{n_{l}}{3}\left(16 a_{4}+\frac{2}{3} a_{1}^{4}+\frac{4}{3} \pi^{2} a_{1}^{2}-\frac{47}{6} \pi^{2} a_{1}+\frac{137}{8} \zeta_{3}-\frac{229}{720} \pi^{4}+\frac{139}{24} \pi^{2}-\frac{2201}{432}\right)-\frac{n_{l}^{2}}{18}\left(7 \zeta_{3}+\frac{19}{6} \pi^{2}+\frac{5767}{432}\right) \\
& +\varepsilon\left[-\frac{224}{3} a_{5}+16 \pi^{2} a_{4}-\frac{5005}{6} a_{4}+\frac{28}{45} a_{1}^{5}+\frac{2}{3} \pi^{2} a_{1}^{4}-\frac{5005}{144} a_{1}^{4}-\frac{88}{27} \pi^{2} a_{1}^{3}-\frac{2}{3} \pi^{4} a_{1}^{2}-\frac{11567}{144} \pi^{2} a_{1}^{2}+14 \pi^{2} \zeta_{3} a_{1}\right. \\
& -\frac{2039}{2160} \pi^{4} a_{1}+\frac{3481}{15} \pi^{2} a_{1}+\frac{125}{8} \zeta_{5}+\frac{29}{32} \zeta_{3}^{2}+\frac{2945}{288} \pi^{2} \zeta_{3}-\frac{348821}{960} \zeta_{3}-\frac{899}{5670} \pi^{6}+\frac{64103}{34560} \pi^{4}-\frac{224592113}{4147200} \pi^{2} \\
& -\frac{2783713}{207360}+\frac{n_{l}}{3}\left(224 a_{5}+\frac{1124}{3} a_{4}-\frac{28}{15} a_{1}^{5}+\frac{281}{18} a_{1}^{4}-\frac{56}{9} \pi^{2} a_{1}^{3}+\frac{281}{9} \pi^{2} a_{1}^{2}-\frac{17}{90} \pi^{4} a_{1}-\frac{644}{9} \pi^{2} a_{1}-\frac{1027}{4} \zeta_{5}\right. \\
& \left.\left.\left.-\frac{119}{16} \pi^{2} \zeta_{3}+\frac{662}{3} \zeta_{3}-\frac{14303}{8640} \pi^{4}+\frac{552083}{13824} \pi^{2}-\frac{153109}{2592}\right)-\frac{n_{l}^{2}}{54}\left(275 \zeta_{3}+\frac{23}{5} \pi^{4}+\frac{1081}{16} \pi^{2}+\frac{253783}{864}\right)\right]+\mathcal{O}\left(\varepsilon^{2}\right)\right\}
\end{aligned}
$$




$$
\begin{aligned}
& +\left(\frac{\alpha}{\pi}\right)^{4}\left[L_{\mathrm{QED}}+\frac{395}{6} a_{5}+28 \pi^{2} a_{4}-\frac{58187}{48} a_{4}-\frac{79}{144} a_{1}^{5}+\frac{7}{6} \pi^{2} a_{1}^{4}-\frac{58187}{1152} a_{1}^{4}-\frac{2411}{216} \pi^{2} a_{1}^{3}-\frac{7}{6} \pi^{4} a_{1}^{2}\right. \\
& -\frac{69311}{576} \pi^{2} a_{1}^{2}+\frac{49}{2} \pi^{2} \zeta_{3} a_{1}-\frac{61}{1728} \pi^{4} a_{1}+\frac{1414153}{3840} \pi^{2} a_{1}-\frac{23093}{128} \zeta_{5}+\frac{203}{128} \zeta_{3}^{2}+\frac{7771}{576} \pi^{2} \zeta_{3}-\frac{327897}{640} \zeta_{3}-\frac{899}{3240} \pi^{6} \\
& +\frac{74911}{69120} \pi^{4}-\frac{148407527}{2073600} \pi^{2}-\frac{778181617}{9953280}-n_{l}(12.18 \pm 0.8) \\
& -n_{l}^{2}\left(\frac{32}{3} a_{5}+\frac{188}{9} a_{4}-\frac{4}{45} a_{1}^{5}+\frac{47}{54} a_{1}^{4}-\frac{8}{27} \pi^{2} a_{1}^{3}+\frac{47}{27} \pi^{2} a_{1}^{2}-\frac{31}{270} \pi^{4} a_{1}-\frac{239}{54} \pi^{2} a_{1}-\frac{601}{48} \zeta_{5}-\frac{\pi^{2}}{2} \zeta_{3}+\frac{6913}{576} \zeta_{3}\right. \\
& \left.\left.-\frac{1297}{51840} \pi^{4}+\frac{25729}{10368} \pi^{2}-\frac{15877}{165888}\right)+\frac{n_{l}^{3}}{216}\left(\frac{467}{2} \zeta_{3}+\frac{71}{20} \pi^{4}+\frac{167}{3} \pi^{2}+\frac{103933}{864}\right)+\mathcal{O}(\varepsilon)\right]+\mathcal{O}\left(\alpha^{5}\right)
\end{aligned}
$$

where $\alpha=\alpha^{\left(n_{f}\right)}(M) ; L_{\mathrm{QED}}=\sum_{i=0,1,2,3, l} L_{i}$ is the $\varepsilon^{0}$ term in $Z_{2}^{(4)}$ of Eq. (26) in [14]. Its numerical value is given in Eq. (15) in this paper.

Numerically, in pure QED $\left(n_{l}=0\right)$ at $\varepsilon=0$ we have

$$
\begin{aligned}
z(M)= & 1-\frac{\alpha}{\pi}-1.09991\left(\frac{\alpha}{\pi}\right)^{2}+4.40502\left(\frac{\alpha}{\pi}\right)^{3} \\
& -2.16215\left(\frac{\alpha}{\pi}\right)^{4}+\mathcal{O}\left(\alpha^{5}\right)
\end{aligned}
$$

where $\alpha=\alpha^{(1)}(M)$, the $\overline{\mathrm{MS}} \mathrm{QED}$ coupling with one active flavor at $\mu=M$, the on-shell electron mass. In contrast to the QCD case (7) the coefficients are numerically smaller and have different signs.

\section{CONCLUSION}

We have calculated the (finite) matching coefficient between the QCD heavy-quark field $Q$ and the corresponding HQET field $h_{v}$ up to four loops. Explicit results are presented for $\mu=M$; results for different values of $\mu$ can be obtained with the help of (known) renormalization group equations. The effect of a nonzero light-flavor mass (e.g., $c$ in $b$-quark HQET) is calculated up to three loops. We also present results for the matching constant in QED.

As a possible application of our results we want to mention the possibility to obtain the QCD heavy-quark propagator (say, in Landau gauge) from lattice QCD results for the HQET propagator. A heavy-quark field can be put onto the lattice only if $M a \ll 1$, where $a$ is the lattice spacing. On the other hand, in HQET simulations there is no lattice $h_{v}$ field at all. The HQET propagator is just a straight Wilson line, i.e., a product of lattice gauge links. It is therefore much easier to obtain the HQET propagator from lattice simulations. After taking the continuum limit, one can get the continuum coordinate-space HQET propagator. Then the QCD heavy-quark propagator can be obtained with the help of the matching coefficient $z(\mu)$, provided that $1 / M^{n}$ corrections can be neglected. Note that this can be done for arbitrarily heavy QCD quark, including the case when the use of the dynamic heavy-quark field on the lattice is impossible.

The main results can be found in the Mathematica files that we provide as Supplementary Material [41].

\section{ACKNOWLEDGMENTS}

We are grateful to R. N. Lee for discussions of the Appendix B. This research was supported by the Deutsche Forschungsgemeinschaft (DFG, German Research Foundation) under Grant No. 396021762TRR 257 "Particle Physics Phenomenology after the Higgs Discovery". This work was supported in part by the EU TMR network SAGEX Marie SkłodowskaCurie Grant agreement No. 764850 and COST action No. CA16201: Unraveling new physics at the LHC through the precision frontier. The work of A. G. was supported by the Russian Ministry of Science and Higher Education. The work of A.S. and V.S. is carried out according to the research program of Moscow Center of Fundamental and Applied Mathematics.

\section{APPENDIX A: THE COUPLING AND GLUON-FIELD DECOUPLING COEFFICIENTS}

The $n_{l}$-flavor QCD strong coupling constant and gauge parameter are related to the corresponding quantities in the $n_{f}$-flavor theory by the decoupling relations

$$
\begin{aligned}
\alpha_{s}^{\left(n_{l}\right)}(\mu) & =\zeta_{\alpha}(\mu) \alpha_{s}^{\left(n_{f}\right)}(\mu) \\
1-\xi^{\left(n_{l}\right)}(\mu) & =\zeta_{A}(\mu)\left[1-\xi^{\left(n_{f}\right)}(\mu)\right]
\end{aligned}
$$

The decoupling coefficients satisfy the renormalization group equations

$$
\begin{aligned}
\frac{d \log \zeta_{\alpha}(\mu)}{d \log \mu}= & 2\left[\beta^{\left(n_{f}\right)}\left(\alpha_{s}^{\left(n_{f}\right)}(\mu)\right)-\beta^{\left(n_{l}\right)}\left(\alpha_{s}^{\left(n_{l}\right)}(\mu)\right)\right], \\
\frac{d \log \zeta_{A}(\mu)}{d \log \mu}= & \gamma_{A}^{\left(n_{f}\right)}\left(\alpha_{S}^{\left(n_{f}\right)}(\mu), \xi^{\left(n_{f}\right)}(\mu)\right) \\
& -\gamma_{A}^{\left(n_{l}\right)}\left(\alpha_{S}^{\left(n_{l}\right)}(\mu), \xi^{\left(n_{l}\right)}(\mu)\right) .
\end{aligned}
$$


It is sufficient to have initial conditions, say, at $\mu=M$ for solving these equations. For the computation of $z(M)$ we need the decoupling coefficients up to $\alpha_{s}^{3} \varepsilon$. Up to the order $\alpha_{s}^{2}$ expression exact in $\varepsilon$ can be found in [42]. The finite three-loop results have been obtained in [43] in term of $N_{c}$ and in [44] for an arbitrary color group. The $\alpha_{s}^{3} \varepsilon$ terms were derived in the course of four-loop calculations [44-46]. However, results for an arbitrary color group, including positive powers of $\varepsilon$, are not explicitly presented in these publications. Therefore, we present them here:

$$
\begin{aligned}
& \zeta_{\alpha}(M)=1-\frac{\alpha_{s}}{\pi} T_{F} n_{h} \frac{\varepsilon}{9}\left(\frac{\pi^{2}}{4}-\zeta_{3} \varepsilon+\frac{3}{160} \pi^{4} \varepsilon^{2}+\mathcal{O}\left(\varepsilon^{3}\right)\right) \\
& -\left(\frac{\alpha_{s}}{\pi}\right)^{2} T_{F} n_{h}\left\{\frac{15}{16} C_{F}-\frac{2}{9} C_{A}+\frac{\varepsilon}{4}\left[\frac{C_{F}}{4}\left(\frac{\pi^{2}}{3}+\frac{31}{2}\right)+\frac{C_{A}}{9}\left(\frac{5}{4} \pi^{2}+\frac{43}{3}\right)\right]\right. \\
& \left.-\varepsilon^{2}\left[\frac{C_{F}}{4}\left(\frac{\zeta_{3}}{3}-\frac{5}{8} \pi^{2}-\frac{223}{16}\right)+\frac{C_{A}}{9}\left(\frac{5}{4} \zeta_{3}+\frac{\pi^{2}}{3}+\frac{523}{72}\right)+\frac{\pi^{4}}{1296} T_{F} n_{h}\right]+\mathcal{O}\left(\varepsilon^{3}\right)\right\} \\
& +\left(\frac{\alpha_{s}}{\pi}\right)^{3} T_{F} n_{h}\left\{\frac{C_{F}^{2}}{3}\left(\pi^{2} a_{1}-\frac{\zeta_{3}}{64}-\frac{5}{8} \pi^{2}-\frac{77}{192}\right)-\frac{C_{F} C_{A}}{6}\left(\pi^{2} a_{1}+\frac{1081}{128} \zeta_{3}-\frac{\pi^{2}}{3}+\frac{8321}{864}\right)\right. \\
& -\frac{C_{A}^{2}}{768}\left(\frac{5}{2} \zeta_{3}-\frac{11347}{27}\right) \\
& -C_{F} T_{F} n_{h}\left(\frac{7}{64} \zeta_{3}+\frac{\pi^{2}}{9}-\frac{695}{648}\right)-\frac{7}{64} C_{A} T_{F} n_{h}\left(\frac{\zeta_{3}}{2}-\frac{35}{81}\right)+\frac{C_{F} T_{F} n_{l}}{18}\left(\pi^{2}+\frac{311}{72}\right)-\frac{C_{A} T_{F} n_{l}}{2592} \\
& -\varepsilon\left[C_{F}^{2}\left(\frac{37}{12} a_{4}+\frac{37}{288} a_{1}^{4}+\frac{251}{288} \pi^{2} a_{1}^{2}-2 \pi^{2} a_{1}+\frac{2759}{576} \zeta_{3}-\frac{241}{3456} \pi^{4}+\frac{439}{384} \pi^{2}+\frac{3329}{3456}\right)\right. \\
& +C_{F} C_{A}\left(\frac{63}{16} a_{4}+\frac{21}{128} a_{1}^{4}-\frac{85}{128} \pi^{2} a_{1}^{2}+\pi^{2} a_{1}+\frac{2413}{512} \zeta_{3}-\frac{1391}{23040} \pi^{4}-\frac{281}{1728} \pi^{2}+\frac{451831}{62208}\right) \\
& -\frac{C_{A}^{2}}{96}\left(263 a_{4}+\frac{263}{24} a_{1}^{4}-\frac{263}{24} \pi^{2} a_{1}^{2}+\frac{27347}{288} \zeta_{3}-\frac{1687}{1440} \pi^{4}-\frac{1063}{216} \pi^{2}-\frac{345115}{1944}\right) \\
& +C_{F} T_{F} n_{h}\left(\frac{3}{4} a_{4}+\frac{a_{1}^{4}}{32}-\frac{\pi^{2}}{32} a_{1}^{2}-\frac{2}{3} \pi^{2} a_{1}+\frac{3353}{1152} \zeta_{3}-\frac{17}{1920} \pi^{4}+\frac{407}{864} \pi^{2}-\frac{67037}{15552}\right) \\
& +\frac{C_{A} T_{F} n_{h}}{8}\left(3 a_{4}+\frac{a_{1}^{4}}{8}-\frac{\pi^{2}}{8} a_{1}^{2}+\frac{1799}{864} \zeta_{3}-\frac{17}{480} \pi^{4}+\frac{113}{1296} \pi^{2}+\frac{1165}{11664}\right) \\
& \left.\left.-\frac{C_{F} T_{F} n_{l}}{9}\left(\zeta_{3}+\frac{403}{192} \pi^{2}+\frac{24911}{864}\right)-\frac{C_{A} T_{F} n_{l}}{27}\left(5 \zeta_{3}+\frac{47}{192} \pi^{2}-\frac{6553}{1728}\right)\right]+\mathcal{O}\left(\varepsilon^{2}\right)\right\}+\mathcal{O}\left(\alpha_{s}^{4}\right) \text {, } \\
& \zeta_{A}(M)=1+\frac{\alpha_{s}}{\pi} T_{F} n_{h} \frac{\varepsilon}{9}\left(\frac{\pi^{2}}{4}-\zeta_{3} \varepsilon+\frac{3}{160} \pi^{4} \varepsilon^{2}+\mathcal{O}\left(\varepsilon^{3}\right)\right) \\
& +\left(\frac{\alpha_{s}}{\pi}\right)^{2} T_{F} n_{h}\left\{\frac{1}{16}\left(15 C_{F}-\frac{13}{12} C_{A}\right)+\frac{\varepsilon}{16}\left[C_{F}\left(\frac{\pi^{2}}{3}+\frac{31}{2}\right)+\frac{C_{A}}{12}\left(5 \pi^{2}+\frac{169}{6}\right)\right]\right. \\
& \left.-\frac{\varepsilon^{2}}{4}\left[C_{F}\left(\frac{\zeta_{3}}{3}-\frac{5}{8} \pi^{2}-\frac{223}{16}\right)+\frac{C_{A}}{12}\left(5 \zeta_{3}-\frac{\pi^{4}}{48}+\frac{13}{24} \pi^{2}+\frac{1765}{144}\right)\right]+\mathcal{O}\left(\varepsilon^{3}\right)\right\} \\
& -\left(\frac{\alpha_{s}}{\pi}\right)^{3} T_{F} n_{h}\left\{\frac{C_{F}^{2}}{3}\left(\pi^{2} a_{1}-\frac{\zeta_{3}}{64}-\frac{5}{8} \pi^{2}-\frac{77}{192}\right)\right. \\
& -C_{F} C_{A}\left(2 a_{4}+\frac{a_{1}^{4}}{12}-\frac{\pi^{2}}{12} a_{1}^{2}+\frac{\pi^{2}}{6} a_{1}+\frac{1765}{768} \zeta_{3}-\frac{11}{720} \pi^{4}-\frac{\pi^{2}}{18}+\frac{15977}{20736}\right) \\
& +C_{A}^{2}\left(a_{4}+\frac{a_{1}^{4}}{24}-\frac{\pi^{2}}{24} a_{1}^{2}+\frac{1805}{4608} \zeta_{3}-\frac{53}{5760} \pi^{4}+\frac{7985}{31104}-\frac{\xi}{48}\left(\zeta_{3}-\frac{677}{144}\right)\right) \\
& -C_{F} T_{F} n_{h}\left(\frac{7}{64} \zeta_{3}+\frac{\pi^{2}}{9}-\frac{695}{648}\right)-\frac{C_{A} T_{F} n_{h}}{144}\left(\frac{287}{8} \zeta_{3}-\frac{605}{27}\right)+\frac{C_{F} T_{F} n_{l}}{18}\left(\pi^{2}+\frac{311}{72}\right)+\frac{C_{A} T_{F} n_{l}}{9}\left(\zeta_{3}-\frac{665}{432}\right)
\end{aligned}
$$




$$
\begin{aligned}
& -\varepsilon\left[C_{F}^{2}\left(\frac{37}{12} a_{4}+\frac{37}{288} a_{1}^{4}+\frac{251}{288} \pi^{2} a_{1}^{2}-2 \pi^{2} a_{1}+\frac{2759}{576} \zeta_{3}-\frac{241}{3456} \pi^{4}+\frac{439}{384} \pi^{2}+\frac{3329}{3456}\right)\right. \\
& +C_{F} C_{A}\left(12 a_{5}+\frac{179}{16} a_{4}-\frac{a_{1}^{5}}{10}+\frac{179}{384} a_{1}^{4}+\frac{\pi^{2}}{6} a_{1}^{3}-\frac{371}{384} \pi^{2} a_{1}^{2}+\frac{17}{120} \pi^{4} a_{1}+\pi^{2} a_{1}-\frac{203}{16} \zeta_{5}+\frac{\pi^{2}}{32} \zeta_{3}+\frac{3141}{512} \zeta_{3}\right. \\
& \left.-\frac{1057}{7680} \pi^{4}-\frac{281}{1728} \pi^{2}+\frac{1199393}{124416}\right) \\
& -C_{A}^{2}\left(6 a_{5}+\frac{611}{96} a_{4}-\frac{a_{1}^{5}}{20}+\frac{611}{2304} a_{1}^{4}+\frac{\pi^{2}}{12} a_{1}^{3}-\frac{611}{2304} \pi^{2} a_{1}^{2}+\frac{17}{240} \pi^{4} a_{1}-\frac{185}{32} \zeta_{5}+\frac{3}{128} \pi^{2} \zeta_{3}+\frac{59395}{27648} \zeta_{3}\right. \\
& \left.-\frac{6679}{138240} \pi^{4}-\frac{10181}{165888} \pi^{2}-\frac{886909}{373248}+\frac{\xi}{96}\left(7 \zeta_{3}+\frac{\pi^{4}}{10}-\frac{233}{576} \pi^{2}-\frac{5737}{144}\right)\right) \\
& +C_{F} T_{F} n_{h}\left(\frac{3}{4} a_{4}+\frac{a_{1}^{4}}{32}-\frac{\pi^{2}}{32} a_{1}^{2}-\frac{2}{3} \pi^{2} a_{1}+\frac{3353}{1152} \zeta_{3}-\frac{17}{1920} \pi^{4}+\frac{113}{216} \pi^{2}-\frac{67037}{15552}\right) \\
& +\frac{C_{A} T_{F} n_{h}}{24}\left(41 a_{4}+\frac{41}{24} a_{1}^{4}-\frac{41}{24} \pi^{2} a_{1}^{2}+\frac{5551}{288} \zeta_{3}-\frac{697}{1440} \pi^{4}-\frac{7}{32} \pi^{2}-\frac{4415}{1944}\right) \\
& \left.\left.-\frac{C_{F} T_{F} n_{l}}{9}\left(\zeta_{3}+\frac{403}{192} \pi^{2}+\frac{24911}{864}\right)-\frac{C_{A} T_{F} n_{l}}{18}\left(\frac{5}{3} \zeta_{3}-\frac{\pi^{4}}{10}+\frac{253}{576} \pi^{2}+\frac{27845}{1296}\right)\right]+\mathcal{O}\left(\varepsilon^{2}\right)\right\}+\mathcal{O}\left(\alpha_{s}^{4}\right)
\end{aligned}
$$

where $\alpha_{s}=\alpha_{s}^{\left(n_{f}\right)}(M), \xi=\xi^{\left(n_{f}\right)}(M)$.

\section{APPENDIX B: ON-SHELL DIAGRAMS WITH TWO MASSES}

Light-quark mass effects in the heavy-quark on-shell propagator diagrams arise for the first time at two loops, see Fig. 1(b). The corresponding integral family can be defined as

$$
\begin{aligned}
& I_{n_{1} n_{2} n_{3} n_{4}}=C \\
& =\frac{C}{\left(i \pi^{d / 2}\right)^{2}} \int \frac{d^{d} k_{1} d^{d} k_{2}}{D_{1}^{n_{1}} D_{2}^{n_{2}} D_{3}^{n_{3}} D_{4}^{n_{4}}}, \quad C=\frac{1}{\Gamma^{2}(1+\varepsilon)}, \\
& D_{1}=M^{2}-\left(p+k_{1}\right)^{2}, \quad D_{2}=-k_{1}^{2}, \\
& D_{3}=m^{2}-k_{2}^{2}, \quad D_{4}=m^{2}-\left(k_{1}-k_{2}\right)^{2}
\end{aligned}
$$

with $p^{2}=M^{2}$. If there are insertions to gluon lines in Fig. 1(b) containing only massless lines, such diagrams are expressed via the integrals (B1) with $n_{2}=n+l \varepsilon$, where $l$ is the total number of loops in these insertions and $n$ is integer ( $n_{1,3,4}$ are always integer). These integrals have been studied in [30]. The IBP algorithm obtained there reduces them to four master integrals

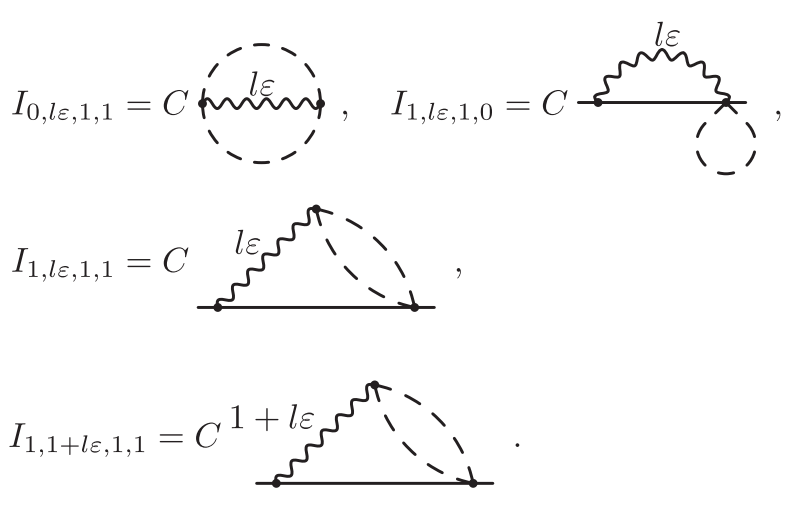

We set $M=1$ and $m=x$.

It is more convenient to use the column vector

$$
j=\left(I_{0, l \varepsilon, 2,2}, I_{2, l \varepsilon, 2,0}, I_{2, l \varepsilon, 2,1}, I_{1, l \varepsilon, 2,2}\right)^{T}
$$

as master integrals instead of (B2). Differentiating them in $m$ and reducing the results back to $j$ [47], we obtain the differential equations

$$
\frac{d j}{d x}=M(\varepsilon, x) j
$$

In many cases such equations can be reduced to an $\varepsilon$-form [48] 


$$
j=T(\varepsilon, x) J, \quad \frac{d J}{d x}=\varepsilon M(x) J .
$$

This makes their iterative solution to any order in $\varepsilon$ almost trivial.

Several terms of small- $x$ and large- $x$ expansions of these integrals (with $l=0$ ) were obtained in [49] using the method of regions (though expressed in a somewhat different language). Differential equations for on-shell sunsets $I_{n_{1}, 0, n_{3}, n_{4}}$ were considered in $[33,50]$, but they were not in $\varepsilon$-form. Several terms of small- $x$ expansions were obtained from differential equations in [51]. However, the easiest way to obtain any finite number of terms in the small- $x$ and large- $x$ expansions is neither the method of regions nor differential equations, but calculating the corresponding residues in the Mellin-Barnes representation [30].

We use the Mathematica package LIBRA [52] which implements the algorithm of [53] to reduce the master integrals $j$ in Eq. (B3) to a canonical basis $J$ :

$$
\begin{aligned}
j_{1}= & I_{0, l \varepsilon, 2,2}=C V_{2,2, l \varepsilon} x^{-2(l+2) \varepsilon}=\frac{2(1-(l+1) \varepsilon)}{(l+2)(1-\varepsilon)} J_{1}, \\
j_{2}= & I_{0, l \varepsilon, 2,0}=C V_{2} M_{2, l \varepsilon} x^{-2 \varepsilon}=\frac{1-2(l+1) \varepsilon}{1-(l+2) \varepsilon} J_{2}, \\
j_{3}= & I_{2, l e, 2,1}=-\frac{1}{2}\left(J_{3}+J_{4}\right), \\
j_{4}= & I_{1, l \varepsilon, 2,2}=\frac{1}{2 x}\left\{-\left[1-2 x-\frac{2 l \varepsilon(1-x)}{1-2 \varepsilon}\right] J_{3}\right. \\
& \left.+\left[1+2 x-\frac{2 l \varepsilon(1+x)}{1-2 \varepsilon}\right] J_{4}\right\},
\end{aligned}
$$

where

$$
\begin{aligned}
& V_{n_{1}}=\overbrace{1}^{1}=\frac{\Gamma\left(\frac{d}{2}-n_{1}\right)}{\Gamma\left(n_{1}\right)}, \\
& M_{n_{1} n_{2} n_{3}}=\underbrace{2}_{1}=H_{0, n_{3}, n_{1}, n_{2}}, \\
& =\frac{\Gamma\left(n_{1}+n_{2}-\frac{d}{2}\right) \Gamma\left(d-n_{1}-2 n_{2}\right)}{\Gamma\left(n_{1}\right) \Gamma\left(d-n_{1}-n_{2}\right)},
\end{aligned}
$$

and [33]

$$
\begin{aligned}
& H_{n_{1} n_{2} n_{3} n_{4}}=\frac{\Gamma}{1}= \\
& \frac{\Gamma\left(n_{1} / 2\right) \Gamma\left(\left(n_{1}-d\right) / 2+n_{2}+n_{3}\right) \Gamma\left(\left(n_{1}-d\right) / 2+n_{2}+n_{4}\right)}{2 \Gamma\left(n_{1}\right) \Gamma\left(n_{3}\right) \Gamma\left(n_{4}\right)} \\
& \times \frac{\Gamma\left(n_{1} / 2+n_{2}+n_{3}+n_{4}-d\right) \Gamma\left(\left(d-n_{1}\right) / 2-n_{2}\right)}{\Gamma\left(n_{1}+2 n_{2}+n_{3}+n_{4}-d\right) \Gamma\left(\left(d-n_{1}\right) / 2\right)}
\end{aligned}
$$

The integrals $J$ satisfy the $\varepsilon$-form differential equations

$$
\frac{d J}{d x}=\varepsilon\left(\frac{M_{0}}{x}+\frac{M_{+1}}{1-x}+\frac{M_{-1}}{1+x}\right) J,
$$

where

$$
\begin{aligned}
& M_{0}=\left(\begin{array}{cccc}
-2(l+2) & 0 & 0 & 0 \\
0 & -2 & 0 & 0 \\
1 & -1 & -(l+2) & l+2 \\
1 & -1 & l+2 & -(l+2)
\end{array}\right) \text {, } \\
& M_{+1}=\left(\begin{array}{cccc}
0 & 0 & 0 & 0 \\
0 & 0 & 0 & 0 \\
1 & -1 & 2 & 2(l+2) \\
0 & 0 & 0 & 0
\end{array}\right) \text {, } \\
& M_{-1}=\left(\begin{array}{cccc}
0 & 0 & 0 & 0 \\
0 & 0 & 0 & 0 \\
0 & 0 & 0 & 0 \\
-1 & 1 & -2(l+2) & -2
\end{array}\right) \text {. }
\end{aligned}
$$

The first two are, of course, known exactly:

$$
\begin{aligned}
J_{1}= & \frac{x^{-2(l+2) \varepsilon}}{(l+1) \varepsilon^{2}} \\
& \times \frac{\Gamma(1-(l+1) \varepsilon) \Gamma^{2}(1+(l+1) \varepsilon) \Gamma(1+(l+2) \varepsilon)}{\Gamma(1-\varepsilon) \Gamma^{2}(1+\varepsilon) \Gamma(1+2(l+1) \varepsilon)}, \\
J_{2}= & \frac{x^{-2 \varepsilon}}{(l+1) \varepsilon^{2}} \frac{\Gamma(1-2(l+1) \varepsilon) \Gamma(1+(l+1) \varepsilon)}{\Gamma(1-(l+2) \varepsilon) \Gamma(1+\varepsilon)} .
\end{aligned}
$$

The equations for $J_{3,4}$ can be solved iteratively in terms of harmonic polylogarithms [54] of $x$. However, we need initial conditions. They can be fixed using the asymptotics of $I_{n_{1} n_{2} n_{3} n_{4}}$ at $x \rightarrow 0$. It is given by contributions of three regions (Sec. III) corresponding to residues of the MellinBarnes representation [30] at three series of poles:

(i) Hard: the poles $s=-n-n_{3}-n_{4}+d / 2(n \geq 0$ is integer), the result is a regular series in $x^{2}$. The leading term is $C G_{n_{3} n_{4}} M_{n_{1}, n_{2}+n_{3}+n_{4}-d / 2}$, where 


$$
\begin{aligned}
& G_{n_{1} n_{2}}=\frac{\Gamma\left(n_{1}+n_{2}-\frac{d}{2}\right) \Gamma\left(\frac{d}{2}-n_{1}\right) \Gamma\left(\frac{d}{2}-n_{2}\right)}{\Gamma\left(n_{1}\right) \Gamma\left(n_{2}\right) \Gamma\left(d-n_{1}-n_{2}\right)} .
\end{aligned}
$$

(ii) Soft-hard: $s=-n-n_{3,4}$. All these poles are double except the first $\left|n_{3}-n_{4}\right|$ ones (and hence, the representation of $I_{n_{1} n_{2} n_{3} n_{4}}$ via hypergeometric functions of $x$ is awkward). We assume $n_{3} \geq n_{4}$, then the result is $x^{d-2 n_{3}}$ times a regular series in $x^{2}$. If $n_{3}>n_{4}$ then the leading term is $C V_{n_{3}} M_{n_{1}, n_{2}+n_{4}} x^{d-2 n_{3}}$; if $n_{3}=n_{4}$ there is an extra factor 2 because each of the lines 3, 4 can be soft.

(iii) Soft: $s=\left(n_{1}-d-n\right) / 2+n_{2}$, the result is $x^{2\left(d-n_{2}-n_{3}-n_{4}\right)-n_{1}}$ times a regular series in $x$. The leading term is $H_{n_{1} n_{2} n_{3} n_{4}} x^{2\left(d-n_{2}-n_{3}-n_{4}\right)-n_{1}}$ (B10). For example,

$$
\begin{aligned}
I_{2, l \varepsilon, 2,1} \rightarrow & \frac{1}{2 \varepsilon^{2}}\left[\frac{1}{l+2} \frac{\Gamma^{2}(1-\varepsilon) \Gamma(1-2(l+2) \varepsilon) \Gamma(1+(l+2) \varepsilon)}{\Gamma(1-2 \varepsilon) \Gamma(1-(l+3) \varepsilon) \Gamma(1+\varepsilon)}-\frac{x^{-2 \varepsilon}}{l+1} \frac{\Gamma(1-2(l+1) \varepsilon) \Gamma(1+(l+1) \varepsilon)}{\Gamma(1-(l+2) \varepsilon) \Gamma(1+\varepsilon)}\right. \\
& \left.+\frac{x^{-2(l+2) \varepsilon}}{(l+1)(l+2)} \frac{\Gamma(1-(l+1) \varepsilon) \Gamma^{2}(1+(l+1) \varepsilon) \Gamma(1+(l+2) \varepsilon)}{\Gamma(1-\varepsilon) \Gamma^{2}(1+\varepsilon) \Gamma(1+2(l+1) \varepsilon)}\right],
\end{aligned}
$$

where the 3 contributions are the hard one $C G_{21} M_{2,1+(l+1) \varepsilon}$ (the pole $s=-1-\varepsilon$ ), the soft-3 one $C V_{2} M_{2,1+l \varepsilon}$ (the pole $s=-1$ ), and the soft one $\mathrm{CH}_{2, l \varepsilon, 2,1} x^{-2(l+2) \varepsilon}$ (the pole $s=-1-(l+1) \varepsilon$ ). The leading asymptotics of $I_{1, l \varepsilon, 2,2}$ is given by the soft contribution $\mathrm{CH}_{1, l \varepsilon, 2,2} x^{-1-2(l+2) \varepsilon}$ (the pole $s=-3 / 2+(l+1) \varepsilon$ ):

$$
\begin{aligned}
I_{1, l \varepsilon, 2,2} \rightarrow & 2^{-1-4(l+2) \varepsilon} \pi^{2} x^{-1-2(l+2) \varepsilon} \frac{1-2(l+1) \varepsilon}{1-2 \varepsilon} \frac{\Gamma(1-\varepsilon) \Gamma(1-2(l+1) \varepsilon)}{\Gamma(1-2 \varepsilon) \Gamma(1-(l+1) \varepsilon)} \\
& \times \frac{\Gamma(1+2(l+1) \varepsilon) \Gamma(1+2(l+2) \varepsilon)}{\Gamma^{2}(1+\varepsilon) \Gamma^{2}(1+(l+1) \varepsilon) \Gamma(1+(l+2) \varepsilon)} .
\end{aligned}
$$

Now we can easily obtain any number of expansion terms of $J_{3,4}$ in $\varepsilon$ for $x<1$ using LIBRA [52]:

$$
\begin{aligned}
J_{3}= & -2\left\{H_{1,0}(x)+H_{0,0}(x)+\frac{\pi^{2}}{3}+\left[-(l+2)\left(2 H_{1,-1,0}(x)+H_{0,1,0}(x)+H_{0,-1,0}(x)\right)+2 H_{1,1,0}(x)\right.\right. \\
& \left.-2(l+3) H_{0,0,0}(x)-l \frac{\pi^{2}}{6} H_{1}(x)-(l+3) \frac{\pi^{2}}{3} H_{0}(x)+\frac{1}{2}(3 l+2) \zeta_{3}-(l+2) \pi^{2} a_{1}\right] \varepsilon \\
& +2\left[(l+2)^{2}\left(-2 H_{1,-1,1,0}(x)+H_{0,1,-1,0}(x)-H_{0,-1,1,0}(x)+H_{0,0,1,0}(x)+H_{0,0,-1,0}(x)\right)\right. \\
& +(l+1)(l+2)\left(H_{1,0,1,0}(x)+H_{1,0,-1,0}(x)\right) \\
& +(l+2)\left(-2 H_{1,1,-1,0}(x)+2 H_{1,-1,-1,0}(x)-H_{0,1,1,0}(x)+H_{0,-1,-1,0}(x)-2 H_{1,0,0,0}(x)\right)+2 H_{1,1,1,0}(x) \\
& +2\left(l^{2}+5 l+7\right) H_{0,0,0,0}(x)+\frac{\pi^{2}}{12}\left[-2 l H_{1,1}(x)-(l+2)(5 l+6)\left(2 H_{1,-1}(x)+H_{0,-1}(x)\right)\right. \\
& \left.+\left(8 l^{2}+23 l+12\right) H_{1,0}(x)+l(l+2) H_{0,1}(x)+\left(6 l^{2}+23 l+24\right) H_{0,0}(x)\right]+\frac{1}{2}(l+3)(3 l+2) \zeta_{3} H_{1}(x) \\
& \left.\left.+(l+2) \pi^{2} a_{1}\left[(l+1) H_{1}(x)+(l+2) H_{0}(x)\right]+(l+2)^{2} \pi^{2} a_{1}^{2}+\left(84 l^{2}+227 l+122\right) \frac{\pi^{4}}{720}\right] \varepsilon^{2}\right\}+\mathcal{O}\left(\varepsilon^{3}\right),
\end{aligned}
$$




$$
\begin{aligned}
J_{4}= & -2\left\{-H_{-1,0}(x)+H_{0,0}(x)-\frac{\pi^{2}}{6}+\left[-(l+2)\left(2 H_{-1,1,0}(x)-H_{0,-1,0}(x)-H_{0,1,0}(x)\right)+2 H_{-1,-1,0}(x)\right.\right. \\
& \left.-2(l+3) H_{0,0,0}(x)-(5 l+6) \frac{\pi^{2}}{6} H_{-1}(x)+(2 l+3) \frac{\pi^{2}}{3} H_{0}(x)+\frac{1}{2}(3 l+2) \zeta_{3}+(l+2) \pi^{2} a_{1}\right] \varepsilon \\
& +2\left[(l+2)^{2}\left(2 H_{-1,1,-1,0}(x)+H_{0,-1,1,0}(x)-H_{0,1,-1,0}(x)-H_{0,0,-1,0}(x)-H_{0,0,1,0}(x)\right)\right. \\
& +(l+1)(l+2)\left(H_{-1,0,-1,0}(x)+H_{-1,0,1,0}(x)\right) \\
& +(l+2)\left(2 H_{-1,-1,1,0}(x)-2 H_{-1,1,1,0}(x)-H_{0,-1,-1,0}(x)+H_{0,1,1,0}(x)+2 H_{-1,0,0,0}(x)\right)-2 H_{-1,-1,-1,0}(x) \\
& +2\left(l^{2}+5 l+7\right) H_{0,0,0,0}(x)+\frac{\pi^{2}}{12}\left[2(5 l+6) H_{-1,-1}(x)+l(l+2)\left(2 H_{-1,1}(x)-H_{0,1}(x)\right)\right. \\
& \left.+\left(4 l^{2}+13 l+12\right) H_{-1,0}(x)+(l+2)(5 l+6) H_{0,-1}(x)-(2 l+3)(3 l+8) H_{0,0}(x)\right]-\frac{1}{2}(l+3)(3 l+2) \zeta_{3} H_{-1}(x) \\
& \left.\left.+(l+2) \pi^{2} a_{1}\left[(l+1) H_{-1}(x)-(l+2) H_{0}(x)\right]-(l+2)^{2} \pi^{2} a_{1}^{2}-\left(36 l^{2}+103 l+58\right) \frac{\pi^{4}}{720}\right] \varepsilon^{2}\right\}+\mathcal{O}\left(\varepsilon^{3}\right) .
\end{aligned}
$$

Up to order $\varepsilon^{1}$ all harmonic polylogarithms can be transformed to logarithms and ordinary polylogarithms up to $\mathrm{Li}_{3}$, e.g., using the Mathematica package HPL [55,56].

Next we consider the case $x>1$. We can rewrite the differential equation (B11) in the form

$$
\frac{d J}{d x^{-1}}=\varepsilon\left(\frac{-M_{0}+M_{+1}-M_{-1}}{x^{-1}}+\frac{M_{+1}}{1-x^{-1}}+\frac{M_{-1}}{1+x^{-1}}\right) J .
$$

It can be solved in terms of harmonic polylogarithms of $x^{-1}$, this is convenient for $x>1$. We use the asymptotics $x \rightarrow+\infty$ for boundary conditions. There are 2 regions:

(i) All lines in (B1) are hard (momenta of order $m$ ). This corresponds to the series of right poles in the
Mellin-Barnes representation $s=n+n_{1}+n_{2}-d / 2$, i.e., to the first term in the hypergeometric representation (A1) in [30], and gives $x^{2\left(d-n_{1}-n_{2}-n_{3}-n_{4}\right)}$ times a regular series in $x^{-2}$. The leading contribution to $I_{n_{1} n_{2} n_{3} n_{4}}$ is $C V_{n_{3}, n_{4}, n_{1}+n_{2}} x^{2\left(d-n_{1}-n_{2}-n_{3}-n_{4}\right)}$.

(ii) Lines 1,2 are soft (momenta of order $M$ ). This corresponds to right poles at $s=n$, i.e., to the second hypergeometric term, and gives $x^{d-2\left(n_{3}+n_{4}\right)}$ times a regular series in $x^{-2}$. The leading asymptotics is $C M_{n_{1} n_{2}} V_{n_{3}+n_{4}} x^{d-2\left(n_{3}+n_{4}\right)}$.

For $I_{2, l \varepsilon, 2,1}$ these two contributions are $\sim x^{-2-2 \varepsilon}$ and $\sim x^{-2-(l+2) \varepsilon}$; for $I_{1, l \varepsilon, 2,2}$ the leading contribution is hard, $\sim x^{-2-(l+1) \varepsilon}$. This information is sufficient for solving the differential equations for $x>1$ using LIBRA [52]:

$$
\begin{aligned}
J_{3}= & -2\left\{-H_{1,0}\left(x^{-1}\right)+\left[-(l+4) H_{0,1,0}\left(x^{-1}\right)-2(l+3) H_{1,0,0}\left(x^{-1}\right)+(l+2)\left(2 H_{1,-1,0}\left(x^{-1}\right)+H_{0,-1,0}\left(x^{-1}\right)\right)\right.\right. \\
& \left.-2 H_{1,1,0}\left(x^{-1}\right)+l \frac{\pi^{2}}{6} H_{1}\left(x^{-1}\right)\right] \varepsilon \\
& +2\left[(l+2)^{2}\left(2 H_{1,-1,1,0}\left(x^{-1}\right)+H_{0,-1,1,0}\left(x^{-1}\right)\right)+(l+2)(l+5) H_{1,0,-1,0}\left(x^{-1}\right)\right. \\
& +(l+2)(l+4)\left(H_{0,1,-1,0}\left(x^{-1}\right)+H_{0,0,-1,0}\left(x^{-1}\right)\right)+(l+2)(l+3)\left(2 H_{1,-1,0,0}\left(x^{-1}\right)+H_{0,-1,0,0}\left(x^{-1}\right)\right) \\
& -(l+3)(l+4) H_{0,1,0,0}\left(x^{-1}\right)-\left(l^{2}+6 l+10\right) H_{0,0,1,0}\left(x^{-1}\right)-\left(l^{2}+5 l+8\right) H_{1,0,1,0}\left(x^{-1}\right) \\
& -2\left(l^{2}+5 l+7\right) H_{1,0,0,0}\left(x^{-1}\right)-(l+4) H_{0,1,1,0}\left(x^{-1}\right)-2(l+3) H_{1,1,0,0}\left(x^{-1}\right) \\
& +(l+2)\left(2 H_{1,1,-1,0}\left(x^{-1}\right)-2 H_{1,-1,-1,0}\left(x^{-1}\right)-H_{0,-1,-1,0}\left(x^{-1}\right)\right)-2 H_{1,1,1,0}\left(x^{-1}\right) \\
& \left.\left.+l \frac{\pi^{2}}{12}\left[(l+4) H_{0,1}\left(x^{-1}\right)-(l+2)\left(2 H_{1,-1}\left(x^{-1}\right)+H_{0,-1}\left(x^{-1}\right)\right)+2 H_{1,1}\left(x^{-1}\right)+H_{1,0}\left(x^{-1}\right)\right]\right] \varepsilon^{2}\right\}+\mathcal{O}\left(\varepsilon^{3}\right),
\end{aligned}
$$




$$
\begin{aligned}
J_{4}= & -2\left\{H_{-1,0}\left(x^{-1}\right)+\left[(l+4) H_{0,-1,0}\left(x^{-1}\right)+2(l+3) H_{-1,0,0}\left(x^{-1}\right)+(l+2)\left(2 H_{-1,1,0}\left(x^{-1}\right)-H_{0,1,0}\left(x^{-1}\right)\right)\right.\right. \\
& \left.-2 H_{-1,-1,0}\left(x^{-1}\right)-l \frac{\pi^{2}}{6} H_{-1}\left(x^{-1}\right)\right] \varepsilon \\
& +2\left[(l+2)^{2}\left(-2 H_{-1,1,-1,0}\left(x^{-1}\right)+H_{0,1,-1,0}\left(x^{-1}\right)\right)+(l+2)(l+5) H_{-1,0,1,0}\left(x^{-1}\right)\right. \\
& +(l+2)(l+4)\left(H_{0,-1,1,0}\left(x^{-1}\right)-H_{0,0,1,0}\left(x^{-1}\right)\right)+(l+2)(l+3)\left(2 H_{-1,1,0,0}\left(x^{-1}\right)-H_{0,1,0,0}\left(x^{-1}\right)\right) \\
& +(l+3)(l+4) H_{0,-1,0,0}\left(x^{-1}\right)+\left(l^{2}+6 l+10\right) H_{0,0,-1,0}\left(x^{-1}\right)-\left(l^{2}+5 l+8\right) H_{-1,0,-1,0}\left(x^{-1}\right) \\
& +2\left(l^{2}+5 l+7\right) H_{-1,0,0,0}\left(x^{-1}\right)-(l+4) H_{0,-1,-1,0}\left(x^{-1}\right)-2(l+3) H_{-1,-1,0,0}\left(x^{-1}\right) \\
& -(l+2)\left(2 H_{-1,-1,1,0}\left(x^{-1}\right)-2 H_{-1,1,1,0}\left(x^{-1}\right)+H_{0,1,1,0}\left(x^{-1}\right)\right)+2 H_{-1,-1,-1,0}\left(x^{-1}\right) \\
& \left.\left.+l \frac{\pi^{2}}{12}\left[-(l+4) H_{0,-1}\left(x^{-1}\right)-(l+2)\left(2 H_{-1,1}\left(x^{-1}\right)-H_{0,1}\left(x^{-1}\right)\right)+2 H_{-1,-1}\left(x^{-1}\right)-H_{-1,0}\left(x^{-1}\right)\right]\right] \varepsilon^{2}\right\} \\
& +\mathcal{O}\left(\varepsilon^{3}\right) .
\end{aligned}
$$

This is, of course, the analytical continuation of (B17) to $x>1$. The same results (B19) can be obtained if we express $J_{3,4}$ via $I_{2, l \varepsilon, 2,1}$ and $I_{1, l \varepsilon, 2,2}$ using (B6) and expand the hypergeometric representations (see Eq. (A1) in [30]) of these two integrals in $\varepsilon$ using the Mathematica package HypExp [57,58]. However, solving the differential equations (B18) up to higher orders in $\varepsilon$ is simpler than expanding hypergeometric functions.

Both (B17) and (B19) lead to identical results at $x=1$ :

$$
\begin{aligned}
J_{3}(1)= & -\frac{\pi^{2}}{3}+\frac{1}{2}(l+2)\left(2 \pi^{2} a_{1}-7 \zeta_{3}\right) \varepsilon \\
& -\left[(l+2)(l+3)\left(8 a_{4}+\frac{1}{3} a_{1}^{4}+\frac{2}{3} \pi^{2} a_{1}^{2}\right)\right. \\
& \left.+\left(17 l^{2}-36 l-124\right) \frac{\pi^{4}}{360}\right] \varepsilon^{2}+\mathcal{O}\left(\varepsilon^{3}\right), \\
J_{4}(1)= & \frac{\pi^{2}}{6}-\frac{1}{2}\left(2 \pi^{2} a_{1}-7 \zeta_{3}\right) \varepsilon \\
& +\left[(l+3)\left(8 a_{4}+\frac{1}{3} a_{1}^{4}+\frac{2}{3} \pi^{2} a_{1}^{2}\right)\right. \\
& \left.+\left(24 l^{2}+27 l-62\right) \frac{\pi^{4}}{360}\right] \varepsilon^{2}+\mathcal{O}\left(\varepsilon^{3}\right) .
\end{aligned}
$$

If $l=0$ and $x=1$, we obviously have $I_{1022}(1)=I_{2021}(1)$, and hence

$$
J_{3}(1)=-2 J_{4}(1)=-4 I_{2021}(1)
$$

Expanding the hypergeometric representation [30] of $I_{2021}$ (or $I_{1022}$ ) at $x=1$ in $\varepsilon$ we get (B20) with $l=0$. Alternatively, we can use another hypergeometric representation $[8,9]$. Using integration by parts we obtain

$$
\begin{aligned}
I_{2021}(1)= & \frac{7}{32 \varepsilon^{2}}\left[\frac{\Gamma(1-\varepsilon) \Gamma^{2}(1+2 \varepsilon) \Gamma(1+3 \varepsilon)}{\Gamma^{2}(1+\varepsilon) \Gamma(1+4 \varepsilon)}-1\right] \\
& +\frac{2^{-2-6 \varepsilon} \pi^{2}}{3} \frac{\Gamma^{3}(1+2 \varepsilon) \Gamma(1+3 \varepsilon)}{\Gamma^{5}(1+\varepsilon) \Gamma^{2}(1+2 \varepsilon)} \\
& +\frac{3}{4} \varepsilon^{2} B_{4}(\varepsilon),
\end{aligned}
$$

where $B_{4}(\varepsilon)$ is given by the formulas (41), (43) in [9]. This leads to the same result.

The functions $L_{\mp}(x)=-\frac{1}{2} J_{3,4}(l=0, \varepsilon=0)$ were used in $[7,8,22,30]$. In addition to the two expressions for these functions in (B17) and (B19), several additional representations can be found in [30].

The results (B17) and (B19) are expansions in $\varepsilon$ where the coefficients are exact functions of $x$. On the other hand, it is straightforward to obtain expansions of $J_{3,4}$ in $x$ (or $x^{-1}$ ) to any finite order using residues of left (or right) poles in the Mellin-Barnes representations of the integrals $j_{3,4}$ (B3), the coefficients being exact functions of $\varepsilon$. If we expand them in $\varepsilon$, they should agree with expansions of (B17) in $x$ and of (B19) in $x^{-1}$. We have checked this up to rather high degrees of $x$ and $x^{-1}$.

Now we can find all contributions to $Z_{j}^{\text {os }}(j=M, Q)$ with the maximum number of quark loops, at most one of which is massive, to all orders exactly in $\varepsilon$ :

$$
\begin{aligned}
Z_{j}^{\mathrm{os}}= & 1+C_{F} \sum_{l} T_{F}^{l-1}\left(n_{0} P\right)^{l-2}\left[n_{0} P B_{j 0}^{(l)}\right. \\
& \left.+(l-1) \sum_{i} B_{j}^{(l)}\left(x_{i}\right)\right]\left(\frac{g_{0}^{2} M^{-2 \varepsilon}}{(4 \pi)^{d / 2}} \Gamma(\varepsilon)\right)^{l}+\cdots,
\end{aligned}
$$

where $g_{0} \equiv g_{0}^{\left(n_{f}\right)}, n_{0}$ is the number of massless flavors, the sum runs over all massive flavors with $x_{i}=m_{i} / M$ (including the external flavor with $x=1$ ) and dots refer to other color structures. Here 


$$
\begin{aligned}
& P=-4 \frac{1-\varepsilon}{(1-2 \varepsilon)(3-2 \varepsilon)} \frac{\Gamma^{2}(1-\varepsilon)}{\Gamma(1-2 \varepsilon)}, \\
& B_{M 0}^{(l)}=-2 \frac{(3-2 \varepsilon)(1-l \varepsilon)}{l(1-(l+1) \varepsilon)(2-(l+1) \varepsilon)} \frac{\Gamma(1+l \varepsilon) \Gamma(1-2 l \varepsilon)}{\Gamma(1+\varepsilon) \Gamma(1-(l+1) \varepsilon)}, \quad B_{Q 0}^{(l)}=B_{M 0}^{(l)}(1+(l-1) \varepsilon), \\
& B_{M}^{(l)}(x)=2 p_{0}\left\{-2 \frac{1-\varepsilon}{l}\left[1-l \varepsilon+l \varepsilon \frac{1-(l-1) \varepsilon}{1+(l-1) \varepsilon} x^{2}\right] J_{1}^{(l-2)}(x)+\left[p_{1}+2 \varepsilon \frac{1+(2 l-3) \varepsilon-(l-1)(l+2) \varepsilon^{2}}{1+(l-1) \varepsilon} x^{2}\right] J_{2}^{(l-2)}(x)\right. \\
& \left.-\left(p_{1}\left(1+x^{2}\right)+p_{2} x\right)(1-x)^{2} J_{3}^{(l-2)}(x)-\left(p_{1}\left(1+x^{2}\right)-p_{2} x\right)(1+x)^{2} J_{4}^{(l-2)}(x)\right\} \text {, } \\
& B_{Q}^{(l)}(x)=p_{0}\left\{-\frac{2 \varepsilon}{l(1-\varepsilon)(1+2(l-1) \varepsilon)(3+2(l-1) \varepsilon)}\right. \\
& \times\left[(1+(l-1) \varepsilon)\left(19 l-3-\left(11 l^{2}+50 l-11\right) \varepsilon-2\left(4 l^{3}-20 l^{2}-15 l+6\right) \varepsilon^{2}+4\left(4 l^{3}-11 l^{2}+2 l+1\right) \varepsilon^{3}-8 l(l-1)^{2} \varepsilon^{4}\right)\right. \\
& \left.+\frac{l(1-\varepsilon)(1+2(l-1) \varepsilon)(3+2(l-1) \varepsilon)}{1+(l-1) \varepsilon}\left(4-(3 l+1) \varepsilon-(l-1)(l-7) \varepsilon^{2}-4(l-1) \varepsilon^{3}\right) x^{2}\right] J_{1}^{(l-2)}(x) \\
& +2\left[2(1-\varepsilon)(1+(l-1) \varepsilon)(1-l \varepsilon)+\varepsilon \frac{4+(11 l-15) \varepsilon-(l-1)(l+17) \varepsilon^{2}-2(l-1)\left(l^{2}-3\right) \varepsilon^{3}}{1+(l-1) \varepsilon} x^{2}\right] J_{2}^{(l-2)}(x) \\
& \left.+\left(p_{3}+p_{4} x+p_{5} x^{2}+p_{6} x^{3}\right)(1-x) J_{3}^{(l-2)}(x)+\left(p_{3}-p_{4} x+p_{5} x^{2}-p_{6} x^{3}\right)(1+x) J_{4}^{(l-2)}(x)\right\},
\end{aligned}
$$

where

$$
\begin{aligned}
p_{0}= & \frac{2 \varepsilon^{2}}{(1-2 \varepsilon)(1-(l+1) \varepsilon)(2-(l+1) \varepsilon)}, \\
p_{1}= & 2(1-\varepsilon)(1-l \varepsilon) \\
p_{2}= & \frac{(1-(l+1) \varepsilon)\left(2+(l-3) \varepsilon-2(l-1) \varepsilon^{2}\right)}{1+(l-1) \varepsilon}, \\
p_{3}= & -4(1-\varepsilon)(1+(l-1) \varepsilon)(1-l \varepsilon), \\
p_{4}= & \frac{2+(3 l-5) \varepsilon-(l-1)(5 l-1) \varepsilon^{2}+4(l-1)^{2} \varepsilon^{3}(2-\varepsilon)}{1+(l-1) \varepsilon} \\
p_{5}= & {\left[2-(5 l+13) \varepsilon+\left(l^{2}-6 l+29\right) \varepsilon^{2}\right.} \\
& \left.+2\left(l^{3}-l^{2}+9 l-13\right) \varepsilon^{3}-8(l-1) \varepsilon^{4}\right] /[1+(l-1) \varepsilon], \\
p_{6}= & 4(1-\varepsilon)(3-2 \varepsilon)(1-l \varepsilon) .
\end{aligned}
$$

The results (B24) at $l=2$ agree with [30] exactly in $\varepsilon$. Note that

$$
\lim _{x \rightarrow 0} B_{M}^{(l)}(x)=B_{M 0}^{(l)} P
$$

so that $Z_{M}^{\text {os }}$ has a smooth limit $x \rightarrow 0$; this is not so for $Z_{Q}^{\mathrm{os}}$
The contribution of these color structures to the ratio of the $\overline{\mathrm{MS}}$ mass and the on-shell one $z_{m}(\mu)=M(\mu) / M$ can be written as

$$
z_{m}(M)=z_{m}^{\left(\beta_{0}\right)}+\sum_{i} \Delta_{m}\left(x_{i}\right)+\cdots,
$$

where $z_{m}^{\left(\beta_{0}\right)}$ is the well-known large- $\beta_{0}$ result [59]

$$
\begin{aligned}
z_{m}^{\left(\beta_{0}\right)} & =1+\frac{1}{2} \int_{0}^{b} \frac{d b}{b}\left(\frac{\gamma_{m}(b)}{b}-\frac{\gamma_{m 0}}{\beta_{0}}\right)+\frac{1}{\beta_{0}} \int_{0}^{\infty} d u S(u) e^{-u / b}, \\
\gamma_{m}(b) & =\frac{2}{3} C_{F} \frac{b}{\beta_{0}} \frac{(3+2 b) \Gamma(4+2 b)}{\Gamma(3+b) \Gamma^{2}(2+b) \Gamma(1-b)}, \\
\gamma_{m 0} & =6 C_{F}, \\
S(u) & =-6 C_{F}\left[e^{(5 / 3) u} \frac{\Gamma(u) \Gamma(1-2 u)}{\Gamma(3-u)}(1-u)-\frac{1}{2 u}\right], \\
b & =\beta_{0} \frac{\alpha_{s}}{4 \pi} \quad\left(\text { where } \alpha_{s} \equiv \alpha_{s}^{\left(n_{f}\right)}(M)\right) .
\end{aligned}
$$

Note that we first expand $S(u)$ in $u$, then integrate term-byterm assuming $\beta_{0}>0$, and at the very end substitute $\beta_{0} \rightarrow-(4 / 3) T_{F} n_{f} . \Delta_{m}(x)$ comes from the differences of diagrams with a single massive quark loop and corresponding diagrams with all quark loops being massless and is given by 


$$
\begin{aligned}
& \Delta_{m}(x)=C_{F} T_{F}\left(\frac{\alpha_{s}}{\pi}\right)^{2}\left\{\frac { 1 } { 2 } \left[(1-x)^{2}\left(1+x+x^{2}\right) H_{1,0}(x)-(1+x)^{2}\left(1-x+x^{2}\right) H_{-1,0}(x)+2 x^{4} H_{0,0}(x)\right.\right. \\
& \left.+x^{2} H_{0}(x)-x\left(3+3 x^{2}-x^{3}\right) \frac{\pi^{2}}{6}+\frac{3}{2} x^{2}\right] \\
& +T_{F} n_{0} \frac{\alpha_{s}}{\pi} \frac{2}{3}\left[(1-x)^{2}\left(1+x+x^{2}\right)\left(H_{1,-1,0}(x)+\frac{\pi^{2}}{12} H_{1}(x)\right)\right. \\
& +(1+x)^{2}\left(1-x+x^{2}\right)\left(H_{-1,1,0}(x)+\frac{5 \pi^{2}}{12} H_{-1}(x)\right)-x\left(1+x^{2}\right)\left(H_{0,1,0}(x)+H_{0,-1,0}(x)+\pi^{2} a_{1}\right) \\
& +x^{4}\left(2 H_{0,0,0}(x)-\frac{13}{6} H_{0,0}(x)-\frac{3}{2} \zeta_{3}\right)-x\left(3+3 x^{2}+x^{3}\right) \frac{\pi^{2}}{6} H_{0}(x)-\frac{1}{12}(1-x)^{2}\left(13+10 x+13 x^{2}\right) H_{1,0}(x) \\
& \left.+\frac{1}{12}(1+x)^{2}\left(13-10 x+13 x^{2}\right) H_{-1,0}(x)+x\left(48-12 x+48 x^{2}-13 x^{3}\right) \frac{\pi^{2}}{72}-\frac{7}{12} x^{2} H_{0}(x)-\frac{11}{8} x^{2}\right] \\
& +\left(T_{F} n_{0} \frac{\alpha_{s}}{\pi}\right)^{2} \frac{2}{3}\left[(1-x)^{2}\left(1+x+x^{2}\right)\right. \\
& \times\left(-2 H_{1,-1,1,0}(x)+H_{1,0,1,0}(x)+H_{1,0,-1,0}(x)-\frac{\pi^{2}}{6}\left(5 H_{1,-1}(x)-4 H_{1,0}(x)\right)+\left(\pi^{2} a_{1}+\frac{3}{2} \zeta_{3}\right) H_{1}(x)\right) \\
& +(1+x)^{2}\left(1-x+x^{2}\right) \\
& \times\left(2 H_{-1,1,-1,0}(x)+H_{-1,0,-1,0}(x)+H_{-1,0,1,0}(x)+\frac{\pi^{2}}{6}\left(H_{-1,1}(x)+2 H_{-1,0}(x)\right)+\left(\pi^{2} a_{1}-\frac{3}{2} \zeta_{3}\right) H_{-1}(x)\right) \\
& +2 x\left(1+x^{2}\right)\left(-H_{0,1,-1,0}(x)+H_{0,-1,1,0}(x)-H_{0,0,1,0}(x)-H_{0,0,-1,0}(x)+\frac{4}{3}\left(H_{0,1,0}(x)+H_{0,-1,0}(x)+\pi^{2} a_{1}\right)\right. \\
& \left.-\frac{\pi^{2}}{12}\left(H_{0,1}(x)-5 H_{0,-1}(x)+6 H_{0,0}(x)\right)-\pi^{2} a_{1} H_{0}(x)-\pi^{2} a_{1}^{2}\right) \\
& +x^{4}\left(4 H_{0,0,0,0}(x)-\frac{13}{3} H_{0,0,0}(x)+\frac{89}{36} H_{0,0}(x)\right) \\
& -\frac{1}{6}(1-x)^{2}\left(13+10 x+13 x^{2}\right)\left(H_{1,-1,0}(x)+\frac{\pi^{2}}{12} H_{1}(x)\right) \\
& -\frac{1}{6}(1+x)^{2}\left(13-10 x+13 x^{2}\right)\left(H_{-1,1,0}(x)+\frac{5 \pi^{2}}{12} H_{-1}(x)\right) \\
& +\frac{1}{72}(1-x)^{2}\left(89+68 x+89 x^{2}\right) H_{1,0}(x)-\frac{1}{72}(1+x)^{2}\left(89-68 x+89 x^{2}\right) H_{-1,0}(x) \\
& +x\left(48+6 x+48 x^{2}+13 x^{3}\right) \frac{\pi^{2}}{36} H_{0}(x)+\frac{47}{72} x^{2} H_{0}(x)+\frac{1}{4} x^{2}\left(6+13 x^{2}\right) \zeta_{3}-x\left(5+5 x^{2}-2 x^{3}\right) \frac{\pi^{4}}{30} \\
& \left.\left.-x\left(330-192 x+330 x^{2}-89 x^{3}\right) \frac{\pi^{2}}{432}+\frac{33}{16} x^{2}\right]+\mathcal{O}\left(\alpha_{s}^{3}\right)\right\}
\end{aligned}
$$

Note that $\Delta_{m}(0)=0$. Expanding the three-loop term in $x$ we reproduce the series (up to $x^{8}$ ) obtained in [31]. The threeloop coefficient exact in $x$ (Fig. 3) and well as the four-loop one are new. The contribution of the external flavor $(m=M)$ is given by

$$
\begin{aligned}
\Delta_{m}(1)= & C_{F} T_{F}\left(\frac{\alpha_{s}}{\pi}\right)^{2}\left[-\frac{\pi^{2}-3}{4}+T_{F} n_{0} \frac{\alpha_{s}}{\pi}\left(\zeta_{3}+\frac{13}{36} \pi^{2}-\frac{11}{12}\right)\right. \\
& \left.-\left(T_{F} n_{0} \frac{\alpha_{s}}{\pi}\right)^{2}\left(\frac{13}{6} \zeta_{3}+\frac{4}{45} \pi^{4}+\frac{53}{216} \pi^{2}-\frac{11}{8}\right)+\mathcal{O}\left(\alpha_{s}^{3}\right)\right]
\end{aligned}
$$




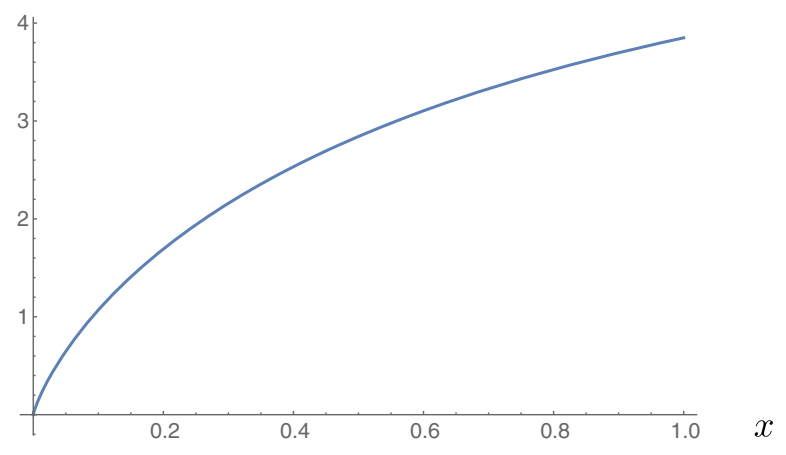

FIG. 3. The coefficient of $\left(\alpha_{s} / \pi\right)^{3} C_{F} T_{F}^{2} n_{0}$ in $\Delta_{m}(x)$.

The three- and four-loop terms here agree with [11] and [12]. We do not present lower-loop terms of $z_{m}$ with positive powers of $\varepsilon$ which may be needed when this ratio is used within calculations containing $1 / \varepsilon$ divergences; these terms can be easily obtained from Eqs. (B23) and (B24).

Contributions to $Z_{h}^{\text {os }}$ with the color structures $C_{F} T_{F}^{l-1} n_{0}^{l-2}$ (i.e., the maximum number of quark loops, one of them is massive with mass $m_{i}$ ) can be calculated using Eq. (B10). The results read

$$
\begin{aligned}
Z_{h}^{\text {os }}= & 1+C_{F} \sum_{l=2}^{\infty} T_{F}^{l-1}\left(n_{0} P\right)^{l-2}(l-1) B_{h}^{(l)} \\
& \times \sum_{i}\left(\frac{g_{0}^{2} m_{i}^{-2 \varepsilon}}{(4 \pi)^{d / 2}} \Gamma(\varepsilon)\right)^{l}+\cdots, \\
B_{h}^{(l)}= & 4 \frac{(3-2 \varepsilon)(1+(l-1) \varepsilon) \Gamma^{2}(1+(l-1) \varepsilon)}{l(l-1)(3+2(l-1) \varepsilon) \Gamma(2-\varepsilon) \Gamma^{2}(1+\varepsilon)} \\
& \times \frac{\Gamma(1-(l-1) \varepsilon) \Gamma(1+l \varepsilon)}{\Gamma(2+2(l-1) \varepsilon)},
\end{aligned}
$$

where $g_{0} \equiv g_{0}^{\left(n_{l}\right)}$ and dots denote other color structures. The $(l=2)$-loop term agrees with [22], and the three-loop one with the corresponding color structure in [33]. According to the regions-based argument in Sec. III,

$$
\lim _{x \rightarrow 0}\left[B_{Q}^{(l)}(x)-B_{Q 0}^{(l)} P-B_{h}^{(l)} x^{-2 l \varepsilon}\right]=0 .
$$

[1] M. Neubert, Heavy-quark symmetry, Phys. Rep. 245, 259 (1994).

[2] A. V. Manohar and M. B. Wise, Heavy Quark Physics, Camb. Monogr. Part. Phys. Nucl. Phys. Cosmol. Vol. 10 (Cambridge University Press, Cambridge, England, 2000).

[3] A. G. Grozin, Heavy Quark Effective Theory, Springer Tracts Mod. Phys. Vol. 201 (Springer, Berlin, 2004), https://doi.org/10.1007/b79301.

[4] F. Bloch and A. Nordsieck, Note on the Radiation Field of the Electron, Phys. Rev. 52, 54 (1937).

[5] A. G. Grozin, Matching heavy-quark fields in QCD and HQET at three loops, Phys. Lett. B 692, 161 (2010).

[6] M. E. Luke and A. V. Manohar, Reparameterisation invariance constraints on heavy particle effective field theories, Phys. Lett. B 286, 348 (1992).

[7] D. J. Broadhurst, N. Gray, and K. Schilcher, Gauge-invariant on-shell $Z_{2}$ in QED, QCD and the effective field theory of a static quark, Z. Phys. C 52, 111 (1991).

[8] D. J. Broadhurst, Three-loop on-shell charge renormalization without integration: $\Lambda_{Q E D}^{M S}$ to four loops, Z. Phys. C 54, 599 (1992).

[9] D. J. Broadhurst, On the enumeration of irreducible k-fold Euler sums and their roles in knot theory and field theory, arXiv:hep-th/9604128.

[10] K. Melnikov and T. van Ritbergen, The three-loop on-shell renormalization of QCD and QED, Nucl. Phys. B591, 515 (2000).

[11] P. Marquard, L. Mihaila, J. H. Piclum, and M. Steinhauser, Relation between the pole and the minimally subtracted mass in dimensional regularization and dimensional reduction to three-loop order, Nucl. Phys. B773, 1 (2007).
[12] R. Lee, P. Marquard, A. V. Smirnov, V. A. Smirnov, and M. Steinhauser, Four-loop corrections with two closed fermion loops to fermion self energies and the lepton anomalous magnetic moment, J. High Energy Phys. 03 (2013) 162.

[13] P. Marquard, A. V. Smirnov, V. A. Smirnov, and M. Steinhauser, Four-loop wave function renormalization in QCD and QED, Phys. Rev. D 97, 054032 (2018).

[14] S. Laporta, High-precision four-loop mass and wave function renormalization in QED, Phys. Lett. B 802, 135264 (2020), arXiv:2001.02739.

[15] S. Laporta, High-precision calculation of the 4-loop contribution to the electron g-2 in QED, Phys. Lett. B 772, 232 (2017).

[16] P. Marquard, A. V. Smirnov, V. A. Smirnov, M. Steinhauser, and D. Wellmann, $(g-2) \mu$ at four loops in QED, EPJ Web Conf. 218, 01004 (2019).

[17] K. G. Chetyrkin and A. Retey, Renormalization and running of quark mass and field in the regularization invariant and MS schemes at three and four loops, Nucl. Phys. B583, 3 (2000).

[18] M. Czakon, The four-loop QCD $\beta$-function and anomalous dimensions, Nucl. Phys. B710, 485 (2005).

[19] T. Luthe, A. Maier, P. Marquard, and Y. Schröder, Five-loop quark mass and field anomalous dimensions for a general gauge group, J. High Energy Phys. 01 (2017) 081.

[20] P. A. Baikov, K. G. Chetyrkin, and J. H. Kühn, Five-loop fermion anomalous dimension for a general gauge group from four-loop massless propagators, J. High Energy Phys. 04 (2017) 119.

[21] K. G. Chetyrkin and A. G. Grozin, Three-loop anomalous dimension of the heavy-light quark current in HQET, Nucl. Phys. B666, 289 (2003). 
[22] D. J. Broadhurst and A. G. Grozin, Matching QCD and heavy-quark effective theory heavy-light currents at two loops and beyond, Phys. Rev. D 52, 4082 (1995).

[23] A. Grozin, J. M. Henn, G. P. Korchemsky, and P. Marquard, The three-loop cusp anomalous dimension in QCD and its supersymmetric extensions, J. High Energy Phys. 01 (2016) 140.

[24] A. Grozin, Leading and next-to-leading large- $n_{f}$ terms in the cusp anomalous dimension and quark-antiquark potential, Proc. Sci., LL2016 (2016) 053.

[25] A. Grozin, Four-loop cusp anomalous dimension in QED, J. High Energy Phys. 06 (2018) 073; J. High Energy Phys.01 (2019) 134(A).

[26] A. Grozin, J. Henn, and M. Stahlhofen, On the Casimir scaling violation in the cusp anomalous dimension at small angle, J. High Energy Phys. 10 (2017) 052.

[27] R. Brüser, A. Grozin, J. M. Henn, and M. Stahlhofen, Matter dependence of the four-loop QCD cusp anomalous dimension: from small angles to all angles, J. High Energy Phys. 05 (2019) 186.

[28] M. Beneke and V. A. Smirnov, Asymptotic expansion of Feynman integrals near threshold, Nucl. Phys. B522, 321 (1998).

[29] V. A. Smirnov, Applied Asymptotic Expansions in Momenta and Masses, Springer Tracts Mod. Phys. Vol. 177 (Springer, Berlin, 2002), https://doi.org/10.1007/3-540-44574-9.

[30] A. I. Davydychev and A. G. Grozin, Effect of $m_{c}$ on $\boldsymbol{b}$ quark chromomagnetic interaction and on-shell two-loop integrals with two masses, Phys. Rev. D 59, 054023 (1999).

[31] S. Bekavac, A. Grozin, D. Seidel, and M. Steinhauser, Light quark mass effects in the on-shell renormalization constants, J. High Energy Phys. 10 (2007) 006.

[32] S. Bekavac, A. G. Grozin, D. Seidel, and V. A. Smirnov, Three-loop on-shell Feynman integrals with two masses, Nucl. Phys. B819, 183 (2009).

[33] A. G. Grozin, A. V. Smirnov, and V. A. Smirnov, Decoupling of heavy quarks in HQET, J. High Energy Phys. 11 (2006) 022, arXiv:hep-ph/0609280.

[34] A. G. Grozin, T. Huber, and D. Maître, On one master integral for three-loop on-shell HQET propagator diagrams with mass, J. High Energy Phys. 07 (2007) 033.

[35] K. Johnson and B. Zumino, Gauge Dependence of the Wave-Function Renormalization Constant in Quantum Electrodynamics, Phys. Rev. Lett. 3, 351 (1959).

[36] B. Zumino, Gauge properties of propagators in quantum electrodynamics, J. Math. Phys. (N.Y.) 1, 1 (1960).

[37] L. D. Landau and I. M. Khalatnikov, The gauge transformation of the green's function for charged particles, Zh. Eksp. Teor. Fiz. 29, 89 (1956), http://www.jetp.ac.ru/ cgi-bin/r/index/r/29/1/p89?a=list [Sov. Phys. JETP 2, 69 (1956)], http://www.jetp.ac.ru/cgi-bin/r/index/e/2/1/p69? $\mathrm{a}=$ list.

[38] E. S. Fradkin, Concerning some general relations of quantum electrodynamics, Zh. Eksp. Teor. Fiz. 29, 258 (1956), http://www.jetp.ac.ru/cgi-bin/r/index/r/29/2/p258?a=list [Sov. Phys. JETP 2, 361 (1956)], http://www.jetp.ac.ru/cgibin/r/index/e/2/2/p361? a=list.
[39] J. Gatheral, Exponentiation of eikonal cross sections in nonabelian gauge theories, Phys. Lett. B 133, 90 (1983).

[40] J. Frenkel and J. Taylor, Non-abelian eikonal exponentiation, Nucl. Phys. B246, 231 (1984).

[41] See Supplemental Material at http://link.aps.org/ supplemental/10.1103/PhysRevD.102.054008 for Mathematica files.

[42] A. Grozin, Decoupling in QED and QCD, Int. J. Mod. Phys. A 28, 1350015 (2013).

[43] K. G. Chetyrkin, B. A. Kniehl, and M. Steinhauser, Decoupling relations to $O\left(\alpha_{s}^{3}\right)$ and their connection to low-energy theorems, Nucl. Phys. B510, 61 (1998).

[44] M. Gerlach, F. Herren, and M. Steinhauser, Wilson coefficients for Higgs boson production and decoupling relations to $\mathcal{O}\left(\alpha_{s}^{3}\right)$, J. High Energy Phys. 11 (2018) 141.

[45] Y. Schröder and M. Steinhauser, Four-loop decoupling relations for the strong coupling, J. High Energy Phys. 01 (2006) 051.

[46] K. G. Chetyrkin, J. H. Kühn, and C. Sturm, QCD decoupling at four loops, Nucl. Phys. B744, 121 (2006).

[47] A. V. Kotikov, Differential equations method. New technique for massive Feynman diagram calculation, Phys. Lett. B 254, 158 (1991).

[48] J. M. Henn, Multiloop Integrals in Dimensional Regularization Made Simple, Phys. Rev. Lett. 110, 251601 (2013).

[49] L. Avdeev and M. Kalmykov, Pole masses of quarks indimensional reduction, Nucl. Phys. B502, 419 (1997).

[50] M. Argeri, P. Mastrolia, and E. Remiddi, The analytic value of the sunrise self-mass with two equal masses and the external invariant equal to the third squared mass, Nucl. Phys. B631, 388 (2002).

[51] A. Onishchenko and O. Veretin, Special case of sunset: Reduction and $\varepsilon$ expansion, Yad. Fiz. 68, 1461 (2005) [Phys. At. Nucl. 68, 1405 (2005)].

[52] R. N. Lee, LIBRA (unpublished).

[53] R. N. Lee, Reducing differential equations for multiloop master integrals, J. High Energy Phys. 04 (2015) 108.

[54] E. Remiddi and J. A. M. Vermaseren, Harmonic Polylogarithms, Int. J. Mod. Phys. A 15, 725 (2000).

[55] D. Maître, HPL, a Mathematica implementation of the harmonic polylogarithms, Comput. Phys. Commun. 174, 222 (2006).

[56] D. Maître, Extension of HPL to complex arguments, Comput. Phys. Commun. 183, 846 (2012), arXiv:hep-ph/ 0703052 .

[57] T. Huber and D. Maître, HypExp, a Mathematica package for expanding hypergeometric functions around integervalued parameters, Comput. Phys. Commun. 175, 122 (2006).

[58] T. Huber and D. Maître, HypExp 2, Expanding hypergeometric functions about half-integer parameters, Comput. Phys. Commun. 178, 755 (2008).

[59] M. Beneke and V.M. Braun, Heavy quark effective theory beyond perturbation theory: renormalons, the pole mass and the residual mass term, Nucl. Phys. B426, 301 (1994). 